\title{
Impact of Design Representations on Creativity of Design Outcomes
}

\author{
Gaetano Cascini ${ }^{\mathrm{a}}$, Lorenzo Fiorineschi ${ }^{b^{*}}$ and Federico Rotini ${ }^{\mathrm{b}}$ \\ ${ }^{a}$ Department of Mechanical Engineering, Politecnico di Milano, Milano, Italy \\ ${ }^{b}$ Department of Industrial Engineering, University of Florence, Firenze, Italy
}

\begin{abstract}
Designers usually spend non-negligible efforts in performing comprehensive design space explorations, but important information exchanged in conceptual design sessions is often lost, even about the rationale behind preferred solutions. As a part of a broader investigation concerning the impact of knowledge coming from previously performed design tasks, this paper describes an experiment aimed at assessing the impact on the design outcomes of two representations used to share existing design information. In particular, the authors compared a function structure, together with a morphological chart, against a hierarchically organized tree of problems and solutions. The design experiment has been performed with a sample composed by 35 engineering students, which have been opportunely subdivided in three groups. The experiment has been structured in three phases and acknowledged literature metrics for assessing idea generation effectiveness have been applied to assess the design outcomes produced by students. Obtained results show that providing information with the two investigated representations leads to potential advantages in terms of Variety of devised concepts. Moreover, additional analysis of the results highlighted that the considered representations led to different idea-generation paths.
\end{abstract}

Keywords: conceptual design; creativity; engineering design; design methods; design information

\section{Introduction}

The continuous evolution of the market demand inevitably leads industries to several design activities devoted to define products totally new or to bring upgrades and/or improvements to the existing ones. In any case, it is easy to infer that a complete accessibility to the knowledge acquired during past design activities is crucial to avoid useless and resources-consuming repetitions. Accordingly, the information about the different design alternatives generated and evaluated in the conceptual design phase, the rationale behind the operated choices and then about the explored design space, should be made comprehensively available.

Textual/graphical technical reports constitute the most diffused way for archiving the recalled set of information but, especially concerning conceptual design activities, it is difficult to collect and reuse data efficiently. In particular, it could be difficult to map the design path that led the designer to the preferred concept. Consequently, if a different designer is asked to improve/upgrade (radically or not) a product previously designed by someone else, he/she inevitably needs to spend resources for identifying and understanding the functionalities, the fundamental working principles and the reasons behind certain decisions.

\footnotetext{
* Corresponding author. Email: lorenzo.fiorineschi@unifi.it. Tel: (+39)055-2758701.
} 
Several conceptual design methods proposed in scientific literature make use of specific representations to explore the design space and to support the ideation process, e.g. those based on Functional Decomposition and Morphology (FDM) (Eppinger \& Ulrich, 2007; Pahl, Beitz, Feldhusen, \& Grote, 2007; Ullman, 2010). Once edited, these representations contain important information about the devised concepts, which are supposed here to be useful for subsequent design activities.

Unfortunately, it is acknowledged that the above mentioned academic methods are not widely diffused in industry (Tomiyama et al., 2009), and consequently designers asked to redesign a product may be unable to apply them comprehensively. Nevertheless, some conceptual design representations could provide useful information to improve the outcomes of re-design tasks, also when involving designers unlearned about the method to which representations belong.

In order to verify the above hypothesis, an experiment has been organized to evaluate the impact of design representations on the outcomes of an idea generation task. Accordingly, the representations from two different conceptual design methods have been considered in this paper as a tool to provide additional information to a sample of engineering students involved in a design task. In particular, representations belonging to the well-known FDM approach and the Problem-Solution Network (PSN) approach (Lorenzo Fiorineschi, Rotini, \& Rissone, 2016) have been considered. Then, by assessing design outcomes through suitable metrics, the objective of the work was to answer to the following key question:

- Do designers get any creative advantage by the availability of design representations from earlier conceptual design activities carried out by others, while performing an individual conceptual design task?

The paper is organized as follows. After a short survey on related works (Section 1) and an introduction to the considered representation models (Section 2), a detailed description of the adopted investigation methodology is reported (Section 3) to describe the experimental settings and the metrics used for performing the assessments. Experimental results are presented in Section 4, while additional investigations are shown in Section 5, to extract more detailed data about the observed behaviours. Discussions are reported in Section 6, together with some considerations about future research activities. Eventually, Conclusions are summarized in Section 7, while the Appendix contains detailed reproductions of the material used for performing the experiment.

\section{Related work}

The concept of "knowledge" is quite complex and could have multiple facets, so that different interpretations can be found in the context of engineering and design, where also different representation models exist (Chandrasegaran et al., 2013). Nevertheless, capturing knowledge during a design process to make it available for subsequent projects is acknowledged to be a fundamental issue in engineering design (Ahmed, 2005), and some methodological proposals can be found accordingly (e.g. Ahmad, Wynn, \& Clarkson (2013), Baxter et al. (2007), Qin, Wang, \& Johnson (2017)).

Although not explicitly conceived for the above-mentioned purposes, schematic representations adopted by systematic conceptual design approaches contain information about the design path followed by designers. Indeed, it is acknowledged in the literature that abstract representations like functional models can be used for storing design information (Atilola, Tomko, \& Linsey, 2015; Medyna, Nonsiri, Coatanéa, $\&$ Bernardb, 2012). More specifically, function structures provided by FDM report detailed information about how the conceived sub-functions interact with each other to allow the implementation of the main functions. Moreover, morphological charts (Pahl et al., 2007) clearly show the investigated working principles and allow to keep track of the combination selected for each overall concept variant. Therefore, information organized according to FDM (or other) representations could be administered to designers involved in re-design activities, to provide a comprehensive framework concerning the underpinnings of the reference product. Moreover, if solution variants explored during previous design tasks are reported, the same representations may provide a comprehensive set of stimuli for designers (e.g. for FDM in form of different working principles for the same function, or even with different sets of sub-functions). 
Specific studies are missing about the reuse of information stored in representations coming from specific conceptual design approaches applied to previous design tasks. Nevertheless, the impact of different stimuli on design outcomes has been deeply investigated and still constitutes an active research topic. Indeed, although designers usually prefer visual stimuli (Gonçalves, Cardoso, \& Badke-Schaub, 2014), other representation types exist and have been investigated in order to assess potential hindrances or aids for idea generation. For example, Atilola et al. (2015) investigated the effect provided by sketches and/or function-trees on design fixation (i.e. the unexpected adherence to a limited set of ideas Jansson and Smith (1991)). Differently, Goldschmidt \& Sever, (2011) evaluated the effect of textual stimuli on idea generation, while Atilola and Linsey (2015) investigated on the impact that different representations (CAD models, photographs and sketches) of a starting example have on fixation and creativity. Moreover Linsey, Wood, and Markman (2008) evaluated the effect of FDM functional structures in a design-by-analogy experiment, concluding that their adoption could enhance design processes as potential support in the search for analogous solutions.

This work fits with this context, but it is specifically focused on investigating the impact of the representations adopted by FDM and PSN conceptual design approaches on the outcomes of a redesign activity. Therefore, an experiment has been performed where designers carried out a redesign activity by starting from previously achieved design outcomes, represented through the formalisms of two different conceptual design methods.

However, because of the scarce diffusion of academic design methods in industry, the authors decided to neglect the effect of the familiarity that designers might have about them. Indeed, the focus of this work is not on the effects (and the flaws) of the methods, but on the effects strictly related to their representations of information. In fact, considering the well-known FDM as a reference, some flaws have been highlighted in the literature (Chakrabarti \& Bligh, 2001; Kroll, 2013), but it is unclear if representations themselves may bring positive or negative effects. Therefore, in a conservative way, the authors considered a sample of designers unlearned about the approaches to which the representations belong.

\section{Considered representations}

In this section, the motivations behind the selection of the two specific representation sets are reported, together with introductory explanations about them. In this way, the authors aim at providing the fundamental information for the understanding of the paper. This introductory overview allows also to add some considerations about the represented information, which leads to some expected limitations of the work.

\subsection{Reasons behind the selection}

Within the context of engineering design methods, it is possible to assert that, among the others, the Systematic Design approach by Pahl and Beitz (Pahl et al., 2007) is one of the most acknowledged ones. Therefore, the related representations used for conceptual design purposes (function structures and morphological charts) constitute an optimal reference for this investigation. Nevertheless, it is strongly based on the concept of function, which is subjected to non-negligible flaws (Eckert, Alink, \& Ruckpaul, 2011; Vermaas \& Eckert, 2013). Indeed, the existence of very different meanings of the term "function" often generates uncertainties and difficulties in mutual communications between designers (Eckert, 2013; Eisenbart, Gericke, \& Blessing, 2013). Accordingly, Pahl and Beitz reported some practical experiences in applying their systematic approach (Pahl et al., 2007), highlighting that thinking in terms of functions is one of the most difficult tasks for designers.

Consequently, the authors decided to consider an additional representation set for this investigation, to evaluate differences between FDM representations (say, "strictly-functional") and other "non-strictlyfunctional" ways for representing concepts. The considered additional representation belongs to the socalled Problem-Solution-Network (PSN) approach (Lorenzo Fiorineschi et al., 2016), which is claimed to 
overcome some FDM flaws highlighted in the literature. The reasons behind the selection of the PSN are twofold. First, the PSN can be considered a comparable alternative to FDM in terms of purpose, but with some non-negligible differences. Indeed, by relying on the co-evolution of problems and solutions, the PSN's representation allows keeping track of the reasons behind certain solutions. However, the underpinning rationale preserves some peculiarities of the FDM approach, e.g. the main design problem decomposition and the morphological composition of different solution variants. Eventually, the PSN has been selected also because the authors have a comprehensive knowledge about the method, allowing them to prepare material for the test, as they did for FDM.

\subsection{FDM representation set}

Well-known design models (e.g. those reported in (Eder \& Hosnedl, 2008; Eppinger \& Ulrich, 2007; Pahl et al., 2007; Ullman, 2010)), consider FDM for performing conceptual design tasks. More precisely, once the overall function of the product is identified according to the set of available requirements, it is decomposed in a set of sub-functions levels, whose number depends on the related complexity (Pahl et al., 2007). The considered functional model follows the well-known Energy-Material-Signal (EMS) formalism (Pahl et al., 2007), where functions are graphically represented by boxes, and specific flows of energy, material and signals (represented by different arrowed lines), constitute inputs and outputs of functions (see Figure 1).

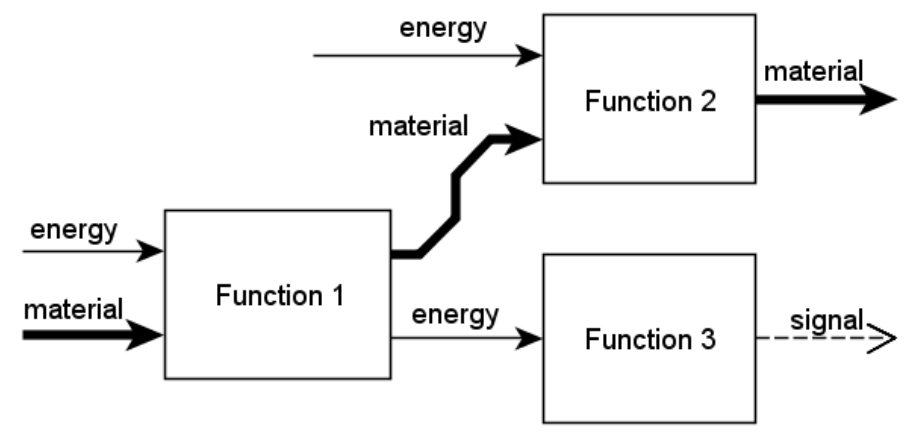

Figure 1. Exemplary EMS functional model

Once a function structure is generated, it constitutes a sort of platform for generating overall concepts. More specifically, different possible solutions are identified (working principles, WP) for the implementation of each sub-function, and their schematic representations are listed in a graphical tool, i.e. the "morphological chart" (Pahl et al., 2007) or "morphological box" (Heller, Schmid, Löwer, \& Feldhusen, 2014), derived from the so-called morphological approach (Zwicky, 1969). In this way, different combinations can be evaluated, within the variety of solutions found for implementing each sub-function (see Figure 2). 


\begin{tabular}{|c|c|c|c|c|}
\hline Functions & 1 & 2 & $\ldots$ & $\mathbf{n}$ \\
\hline Function 1 & WP 1.1 & WP 1.2 & $\ldots$ & WP 1n \\
\hline Function 2 & WP $7: 1$ & WP 2.2 & $-=-m$ & \\
\hline Function 3 & NP 3.1 & WP 3.2 & $\cdots$ & \\
\hline
\end{tabular}

Figure 2. Generic morphological chart associated with the function structure represented in Figure 1, and related combinations of solution variants.

\subsection{PSN representation set}

PSN is a systematic approach conceived to support the designer during the conceptual stage. It is subdivided into three main phases, i.e. the Concept Generation, the Concept Composition and the Concept Selection (Lorenzo Fiorineschi et al., 2016). In the first phase, the overall design problem is decomposed in a network of problems and solutions, hierarchically organized according to specific levels of abstraction. More in particular, different solution variants can be generated for each problem, each of them potentially generating one or more additional sub-problems to be solved, and so forth. Referring to Figure 3 problems $(\mathrm{Pb})$ are represented by yellow boxes, while solutions ( $\mathrm{S}$ ) with green boxes, where textual and (if necessary) pictorial information are reported. The PSN approach considers as a problem any task-related question that can be proposed in the form: "How to verb - noun?", thus generally including problems concerning functions, system qualities, properties or performances.

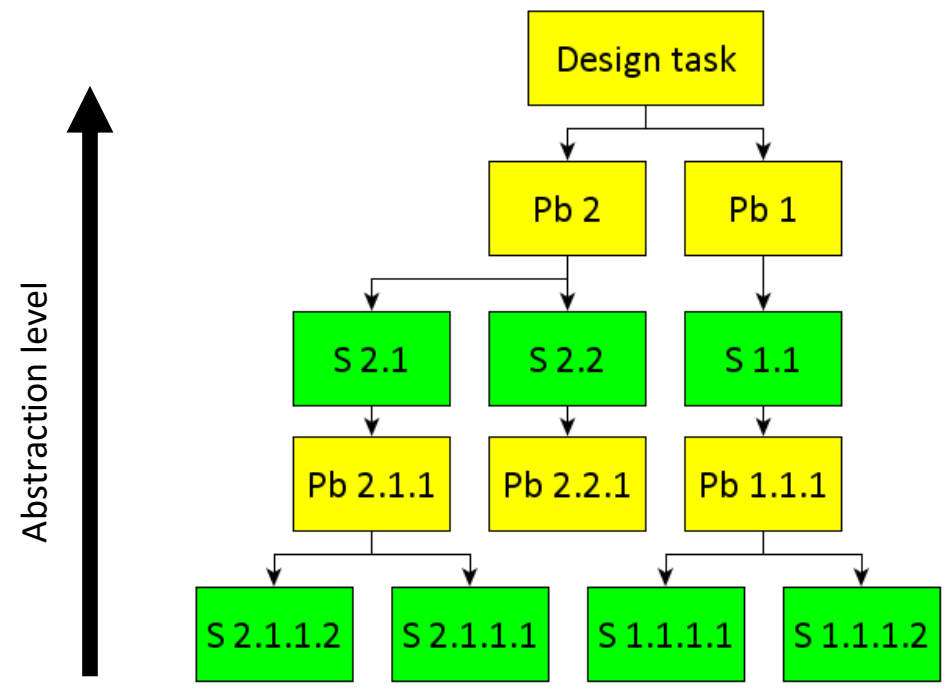

Figure 3. Problem-Solution Network 
The network is built by following a set of six rules, which provide indications about how to proceed with the formulation of problems and the related solutions (for more detailed information refer to Fiorineschi et al. (2016)).

In the second phase, different solutions combinations (i.e. different PSN ramifications) are evaluated to obtain different overall concept variants (Figure 4). In this step, the network acts as a morphological tool where different solution variants are listed for each problem, by following a problem-solution coevolutionary relationship from higher to lower abstraction levels (Figure 3).
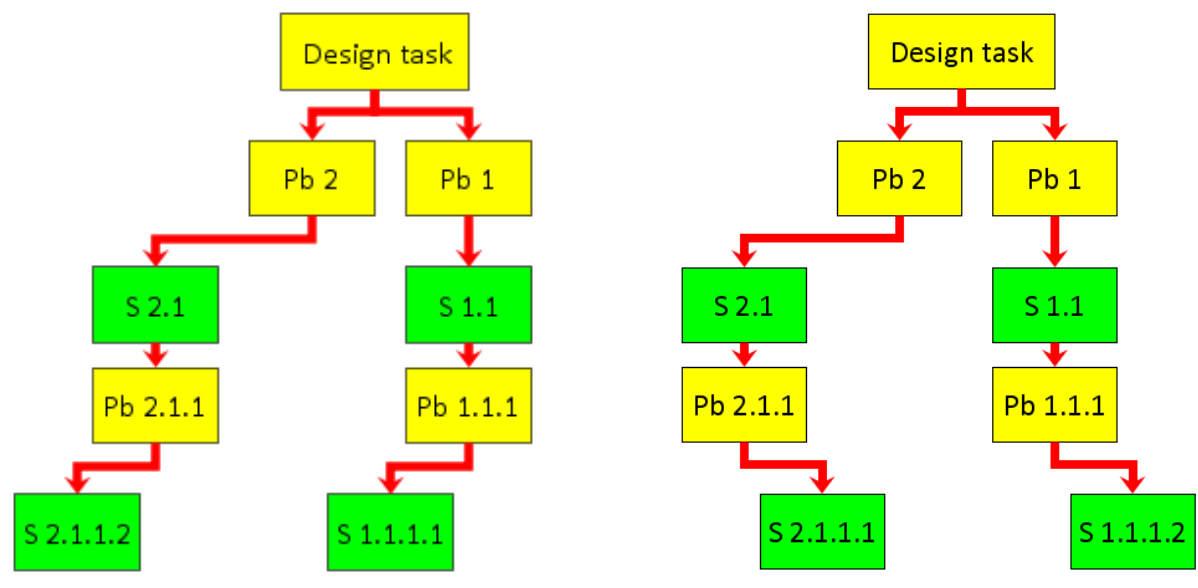

Figure 4. Different morphological compositions of PSN branches from the generic example of Figure 3. Solution $\mathrm{S}-2.2$ in Figure 3 cannot be considered since leading to the unsolved problem $\mathrm{Pb}-2.2 .1$.

Eventually, similarly to FDM, concept variants are selected through classical processes acknowledged by literature, e.g. the "Concept selection matrix" (Pugh 1991), "Selection charts" (Pahl, et al. 2007) or QFDlike matrices (Akao 1990).

\subsection{Limitations imposed for this work}

As shortly introduced in the previous paragraphs, both PSN and FDM representations allow keeping track of the different solution variants for each single problem or function to be implemented. Therefore, they offer the opportunity of reusing also design efforts spent on un-preferred solutions to provide potential design stimuli in future design activities. However, such a potential is not investigated here since the paper is focused only on the representation of a single concept (i.e. a single set of ramification in the PSN and a single EMS structure equipped with a single-column morphological chart). The reason behind this decision lies in the different expansion that the two considered representations may undergo, depending on the concept variants to be represented. More precisely, while PSN allows adding an indefinite number of additional ramifications on the same network (mainly expanding it horizontally), independently on the way the sub-functions interact each other, FDM may need the realization of a different number of function structures, each of them linked to a specific morphological chart. Indeed, different concepts may consider different EMS flow variants (e.g. different energy sources), potentially leading to different sub-function and then different function structures.

Probably, the differences in the representation's expansion somehow affect designers and their design outcomes but, de facto, it is an additional variable that should be investigated with additional experiments. Differently, the causes of possible observed effects may be unclear.

Moreover, both the methods foresee an evaluation phase where important information about decisions behind concept selection is registered in matrices or charts (e.g. Pugh (1991) or Eppinger \& Ulrich (2007)). 
Nevertheless, the recalled information is not considered in this paper, whose focus is on the effects that can be observed by providing information about the fundamentals of product concepts.

\section{Investigation method}

The following section details the investigation method by describing the sample of convenience, the testing procedure and the metrics adopted for performing the required assessments.

\subsection{Sample of convenience}

The investigation has been performed thanks to the participation of 35 Master of Science (MS) students enrolled in a Mechanical Engineering study programme, 2nd year. The sample was almost entirely composed by male subjects (only two females), and by thirteen foreign students (approximately $37 \%$ ).

The design experiment described hereafter was proposed to students just a couple of weeks after the start of the semester, before introducing FDM and PSN, as well as any model to represent design information.

In order to provide the basic and fundamental information needed to perform the required activities, a short explanation of the two modelling schemes (30 minutes overall) has been proposed, before starting the testing procedure.

The sample of students has been subdivided into three different groups, two of them devoted to the exploitation of one of the seòected representations (namely PSN and FDM groups), while the third group (EXP group) was considered as a reference, by administering a technical drawing showing an exploded view of the starting product. In this way, a sort of "placebo" has been administered to the third group, providing only low-informative representation of previous design activities.

Since the sample was not so numerous, the authors opted for making the students work individually to collect more relevant data. For organizational issues, students have been divided as follows: 13 students in the EXP group, 10 and 12 in the FDM and PSN groups respectively.

\subsection{Testing procedure}

In order to evaluate the effects of the two representation schemes, the testing procedure was organized in three distinct phases (see Table 1) where students were asked to perform the same specific design task, with an increasing amount of information.

Table 1. The three test phases and the information/stimulus available to students.

\begin{tabular}{ccccc} 
& $\begin{array}{l}\text { Initial pen } \\
\text { description }\end{array}$ & Task description & $\begin{array}{l}\text { Investigated } \\
\text { representations }\end{array}$ & $\begin{array}{l}\text { Additional verbal } \\
\text { stimulus }\end{array}$ \\
\hline Phase 1 & $\mathrm{X}$ & $\mathrm{X}$ & & \\
Phase 2 & $\mathrm{X}$ & $\mathrm{X}$ & $\mathrm{X}$ & $\mathrm{X}$ \\
Phase 3 & $\mathrm{X}$ & $\mathrm{X}$ & $\mathrm{X}$ & $\mathrm{X}$ \\
\hline
\end{tabular}

Phase 1 was intended to provide initial and neutral information about the considered sample of convenience. More specifically, the authors expected to extract sufficient data to characterize each group (according to specific metrics), to subsequently assess the impact derived from the introduction (in the second phase) of the investigated representations. Accordingly, in the first phase, the same material has been provided to all groups, containing the textual and graphical descriptions of the design task (Figure 5), together with a short textual description of a common ball-point pen, i.e. the subject of the design task (Figure 6). 
DESIGN TASK DESCRIPTION

Imagine you are working in a firm producing ball pens, like the one in the following picture:

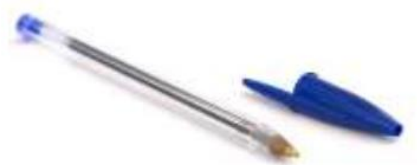

You are asked to find new potential solutions for pens suitable to write upside-down.

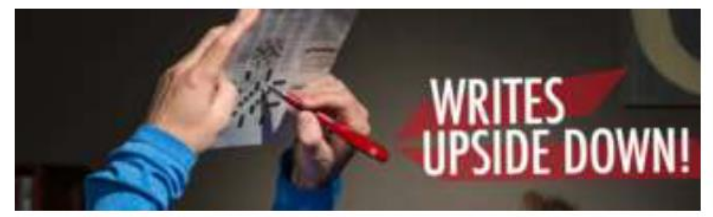

For the scope of this exercise, do not consider economic constraints and try to generate as many solutions as you can. Please consider the following indications:

1. Read/observe the starting material that we provide. The exercise is subdivided in three stages, each one characterized by different reference materials and additional indications.

2. Generate solutions in each stage, trying to propose different concepts with respect to those already available in the market.

3. Aim at the largest variety of your concepts

4. Provide details about your concepts, so as to make it clear what is their working principle and their expected viability

\section{Enjoy!}

Figure 5. Indications provided with the design task description 


\section{Textual description of a ball pen}

A ballpoint pen is a writing instrument which features a tip that is automatically refreshed with ink. It consists of a precisely formed metal ball seated in a socket below a reservoir of ink. As the pen is moved along a writing surface, ink is delivered. Even though ballpoint pens were first patented in the late nineteenth century, they only started to reach commercial significance in the early 1950s. Now, ballpoint pens dominate the writing instrument market, selling over one hundred million pens each year worldwide.

The ballpoint pen was developed as a solution to the problems related to writing with a fountain pen. Fountain pens require the user to constantly refresh the pen by dipping its tip in ink. This is not necessary with a ballpoint pen because it is designed with its own ink reservoir, which uses capillary action to keep the ink from leaking out. At the tip of the pen is a freely rotating ball seated in a socket. Only part of this ball is exposed; the rest of it is on the inside of the pen and is constantly being bathed by ink from the reservoir. Pressing the tip of the pen on the writing surface causes the ball to roll. This rolling action then transfers ink from the inside of the pen to the writing surface.

While different designs of ballpoint pens are available, many of the components are the same. Common components include a ball, a point, ink, an ink reservoir or cartridge, and an outer housing. Some pens are topped with a cap to prevent it from leaking or having its point damaged.

Figure 6. Textual description of a ball-point pen administered together with the design task description.

The design task was focused on the problem of "writing upside-down", arising from the negative action of Gravity on the fluid ink when the pen is overturned. The authors selected this specific task since they assumed that each participant had the same familiarity with pens and with this problem. Moreover, the authors also assumed that the difficulty level of the task matches the mean technical expertise level of the sample, and complies with the available testing time. In this first round, participants were asked to conceive the highest number of ideas, by providing both graphical sketches and short textual descriptions of the generated concepts. For this purpose, an "unlimited" set of paper forms have been provided, where students could record their ideas. The time dedicated for idea generation was 25 minutes, which is consistent with the evidence that productivity of brainstorming-based design session decreases after half an hour, while the largest amount of best ideas are generated during the first 15 minutes (Howard, Dekoninck, \& Culley, 2010).

In the second test phase, each student received an additional paper sheet with the information structured according to the representation scheme assigned to the group. Therefore, students belonging to the EXP group received the exploded-view drawing and the bill of material of a common ball-point pen. Students of the FDM group received a representation scheme describing how the ball pen works based on function structure and a single-column morphological chart. Similarly, students belonging to the PSN group received a network of problems and solutions describing the current design of the ball pen. An overall view of the different information sheets provided to the three groups of treatment is depicted in Figure 7, but it can be found with better readability in the Appendix. 


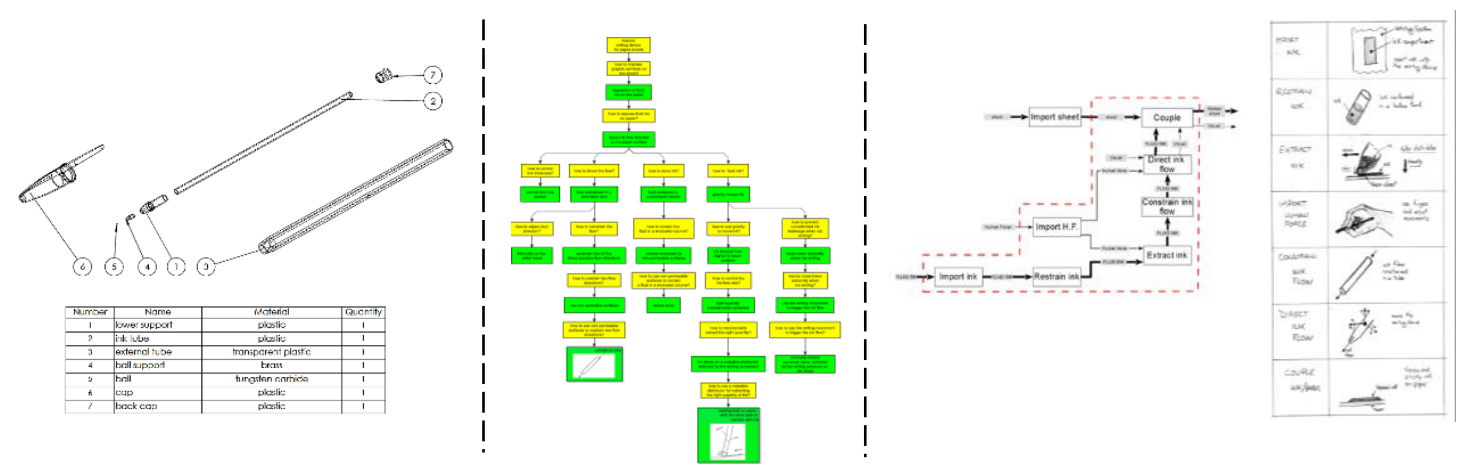

Figure 7. Overall view of the supplemental material provided in phase 2, respectively (from the left) for EXP, PSN and FDM groups (see Appendix for full readability).

Each student was asked to conceive additional ideas as much as they could, but trying to exploit the additional information provided with the sheet received. Also in the second phase, the time was limited to 25 minutes, still with virtually unlimited forms for representing the generated concepts.

Furthermore, a third phase has been considered for extracting additional information. More precisely, at the end of the second phase, the compiled forms have been collected, while leaving the representations administered in Phase 2, and providing the following verbal suggestion to students: "try to conceive concepts not exploiting the ball-tip". The authors decided to carry out this further experiment to understand if different reactions can be observed after the introduction of a verbal stimulus that explicitly invites the testers to exclude the specific structural detail characterizing the starting solution. Indeed, besides graphical and textual representations, it is also quite common to share pieces of information verbally among designers, even during idea generation processes (Atilola \& Linsey, 2015; Atilola et al., 2015), and sometimes they might act as creativity triggers. Also the third phase lasted 25 minutes.

\subsection{Evaluation procedure}

In order to evaluate the ideas produced by students, and then the impact of the different representation schemes on design outcomes, the authors referred to acknowledged literature metrics. More precisely, those based on the assessment of the quantity, quality, variety and novelty of ideas (Shah, Vargas-Hernandez, \& Smith, 2003) have been considered here for a unique overall function of the system, i.e. "impressing symbols on a paper sheet". Moreover, to verify the statistical reliability of the differences emerged in the results, (when possible) the confidence intervals (CI) of the obtained distributions (Sheskin, 2003) have been checked. Indeed, such a rough approach is suitable for the limited sample. In addition, t-tests (Sheskin, 2003) have been performed within each group (when possible), but the authors are conscious that for samples with less than 20 subjects, results must be interpreted with caution. In these cases, non-parametric statistical approaches such as the Mann-Whitney one (Ross, 2009) should be preferred.

At the end of the test, participants sketched a total number of 231 ideas, two of which are shown in Figure 8 with the aim to provide just some examples to the reader. Besides sketches, students provided short textual descriptions of solutions to explain the functioning of each proposed idea. 


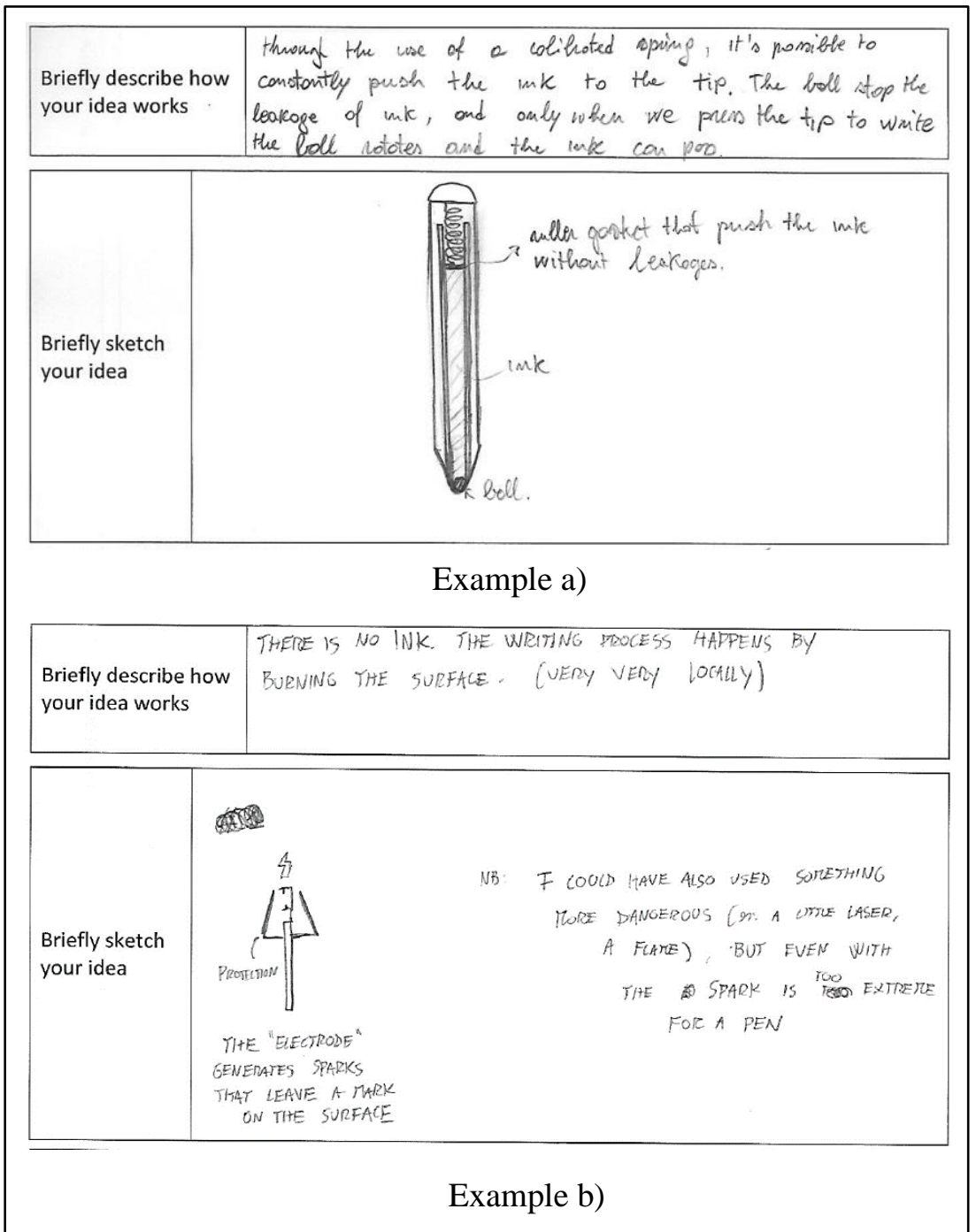

Figure 8. Two examples from the set of ideas produced by participants

The following paragraphs report an introduction to each of the considered metrics.

\subsubsection{Quantity}

For assessing this parameter related to subjects' fluency, the number of conceived ideas have been counted for each student, in each group, and in each phase. Then, the "mean quantity per group" (Qn) has been calculated, for each group and each phase, by referring to Equation 1:

$$
Q n=\frac{n_{i d}}{n_{s t}}
$$

where, " $n_{i d}$ " represents the total number of ideas conceived by a specific group in a specific phase, and $" n_{s t}$ " represents the number of students for the considered group. This metric has been used in place of the "total number" ideas (as suggested in Shah et al. (2003)), because of the different size of the three groups. In fact, by means of $Q n$, Quantity can be evaluated independently from the actual number of participants. 


\subsubsection{Quality}

Since the students generated their concepts without a detailed design specification, the comprehensive verification of the compliance with the design requirements was not possible. Therefore, the quality of ideas proposed by participants has been assessed by judging the technical feasibility according to the three-levels scale proposed by Linsey et al. (2011) (see Figure 9). This metric is well suited for the detail level of the concepts proposed by students.

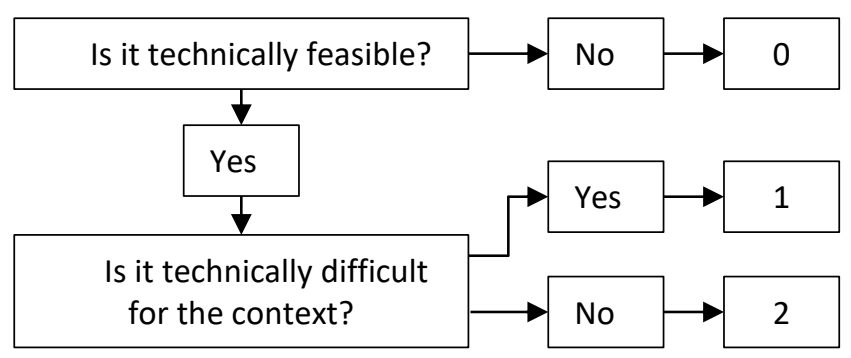

Figure 9. Adopted quality scale (from Linsey et al. (2011))

Quality scores were assigned to the concepts through a conjoint evaluation session performed by the authors. Moreover, since quality assessments are often affected by subjectivity (especially when only little information is present) an Inter-Rater Reliability (IRR) test has been performed by evaluating the Cohen's Kappa (Sheskin, 2003) on scores assessed by an additional evaluator. In particular, the latter performed the assessment on a randomly selected subset (35\%) of concepts generated by students.

In order to provide a reference for the reader, it is worth to notice that the two examples shown in Figure 8 have been assessed with the highest and the lowest QL levels (examples "a" and "b" respectively). In the first case, according to the poorly detailed representation, no technical problem has been found for developing and/or using a pen based on that concept.

For the second idea, instead, many doubts may arise concerning the actual viability, considering that it can be very difficult to realize an "electrode" pen for paper sheets a $(\mathrm{QL}=0)$.

\subsubsection{Variety}

This parameter has been assessed as originally proposed in Shah et al. (2003), considering a single function (deposing ink) and a single design stage (conceptual), but without considering the lowest hierarchical level, i.e. detail variations. Such a decision is a direct consequence of the limited detail level of the conceptual solutions obtained from the test.

Therefore, Equation 2 has been used for calculating the Variety score of each group for each testing phases:

$$
V=\frac{10 \cdot \mathrm{n}_{p p}+6 \cdot \mathrm{n}_{w p}+3 \cdot n_{s t r}}{n_{i d}}
$$

where, " $n_{i d}$ " is the total number of ideas for the specific group in the specific testing phase, " $n_{p p}$ " is the number of the observed physical principles variants, " $n_{w p}$ " is the number of the observed working principles variants, and $n_{s t r}$ " is the number of the observed main structure variants. Note that the authors 
intentionally mention "structure variants" instead of "embodiment variants", because only the conceptual design phase is actually considered in this work.

Similarly to what suggested by Vargas Hernandez, Okudan, \& Schmidt (2012), the entire set of ideas from all groups and all phases has been merged to identify a common set physical principle (PP), working principle (WP) or structural (STR). Moreover, the item variants (for PP, WP and STR) were assigned to the concepts through a conjoint evaluation session performed by the authors.

Subsequently, the Variety assessment has been performed for each group separately. It is important to notice that for Variety it is not possible to evaluate the confidence intervals and to perform t-tests since the number of variants for each item is extracted for each group in each phase as a whole, and not comparing the number of different variants considered by each student.

To provide an example to the reader, the items assigned to the concepts shown in Figure 8 are reported here. More specifically, Concept "a" has been coded with the physical principle "liquid in pressure", the working principle "moving surface" and the main structural characteristic "coaxial spring". Differently, Concept "b" has been coded with physical principle "heating", working principle "spark", and main structural characteristic "sparkling-tip".

\subsubsection{Novelty}

The "a-posteriori" knowledge approach has been adopted as a reference in this paper although with some differences with the original version proposed by Shah and Vargas-hernandez (2003). In particular, Shah and Vargas-hernandez refer to the identification of common "key attributes" across the entire set of ideas to identify how they have been implemented in each idea. Moreover, they also assign different weights for taking into consideration the related importance levels. However, the identification of such a set of common attributes was extremely difficult in this experiment due to the high number of examined ideas and the related high heterogeneity.

To overcome such a problem, the authors took inspiration from literature metrics considering Varietyrelated parameters for assessing Novelty. Indeed, Vargas Hernandez, Okudan, \& Schmidt (2012) proposed to use genealogy trees from Variety assessments for counting the ideas with each WP, and then calculating Novelty in relation to the number of branches. However, according to Johnson, Caldwell, Cheeley, \& Green (2016), in this way siblings with the same WP receive the same score, then neglecting part of the assessment. Differently, Peeters, Verhaegen, Vandevenne, \& Duflou (2010) proposed to use PP, WP and Embodiment characteristics stored in the genealogy tree for assessing Novelty. In this paper, the authors mainly refer to the latter metric, but as already explained for the Variety metric, instead of the "Embodiment" level, the STR level has been taken into account, i.e. the structural details characterizing the concept and that can be observed in the rough sketches carried out by students. However, the difference between Embodiment and STR is quite vague, as well as the border between Conceptual Design and Embodiment Design.

Therefore, different weights have been assigned to different items (i.e. PP, WP or STR) where the differences have been observed. As a consequence, referring to Shah and Vargas-Hernandez (2003), the "aposteriori" novelty score has been calculated for each item (PP, WP and STR) and for each group in each phase (Equation 3).

$$
N_{i}=10 \cdot \frac{\mathrm{n}_{i d}-\mathrm{I}}{n_{i d}}
$$

where " $N_{i}$ " is the novelty score associated to a specific item "i" (PP, WP or STR) for a specific group in a specific phase," $n_{i d}$ " is the total number of ideas, and "I" is the count of the occurrences of the specific item variant. In a few words, PP, WP and STR substitute the general key attributes of the original metric.

For what concerns the universes of solutions (i.e. the set of PP, WP and STR variants) used for assessing novelty of each idea, they have been considered independent between groups of students (EXP, FDM and PSN). Moreover, universes have been considered as incrementally expanding from the first to the third phase (L. Fiorineschi, Frillici, \& Rotini, 2018). Therefore, for each group (independently from each other), 
the novelty score of each idea is assessed by taking into consideration not only the universe of the current phase but also the precedent ones (when present).

Then, the novelty scores for each idea have been assessed with Equation 4, by taking into consideration the novelty scores of the items characterizing the solution (as previously identified in the Variety assessment), and the related weights. The latter has been obtained by the normalization of the three weights considered in Equation 3, i.e. 10 for PP, 6 for WP and 3 for STR.

$$
\mathrm{N}=0,53 \cdot N_{p p}+0,32 \cdot N_{w p}+0,16 \cdot N_{s t r}
$$

Eventually, for each group and for each testing phase the "mean novelty" has been calculated, i.e. the mean value of the novelty calculated across all ideas within a group and within a specific testing phase, to observe the overall behaviour of the investigated sample of convenience. More precisely, the mean score for each group in each phase has been determined by considering only the novelty scores of the related ideas.

For the sake of clarity, two illustrative examples of novelty assessments are reported here in the following by referring to Figure 8 , whose results are shown in Table 2 . The physical principle used in Example "a" has been proposed quite often within the specific group, and due to the weights introduced in Section 3, it leads to a low novelty score. Differently, Example " $b$ " is based on extremely original items (for the specific group and phase), and then, a relatively high novelty score has been assigned.

Table 2. Novelty assessment for examples of Figure 8.

\begin{tabular}{lll}
\hline Example & $\mathrm{A}$ & $\mathrm{b}$ \\
\hline Physical principle score & 2,50 & 9,63 \\
Working principle score & 6,56 & 9,88 \\
Embodiment variants & 8,44 & 9,88 \\
score & 4,72 & 9,75 \\
Novelty score & \\
\hline
\end{tabular}

\section{Results}

Hereinafter, the results of the assessment are reported.

\subsection{Quantity}

Overall, the three groups behaved quite differently in the first round and revealed an unexpected heterogeneity. For all groups, a reduction of the mean quantity of generated ideas has been observed from the first to the second phase, while from the second to the third one, only FDM and PSN show a slight growth. However, as shown in Figure 10, it is possible to observe that especially for the FDM group, CIs are highly overlapping among subsequent test phases. Table 3 shows the results of the performed paired ttests, which confirm that the FDM group is affected by higher data dispersion.

Due to the limited extension of the considered sample, where less than 20 participants constitute the groups, a Mann-Whitney test has been performed to further verify if meaningful differences exist between the three phases. As a result, it emerged that for the EXP group there is not statistically reliable difference between the medians of Phase 1 and Phase 2 (p-value $<0,05$ ). Therefore, the EXP results shown in Table 3 should be considered with care.

Besides, the higher and the most reliable reduction from the first to the second phase was recorded for the group that generated the biggest amount of concepts in the first round (PSN). Nevertheless, the same group is the sole that shows a growth from Phase 2 to Phase 3 nearly statistically reliable (according to the reference p-value). However, while the additional Mann-Whitney test shows that there is a statistically 
reliable difference between the medians of Phase 1 and Phase 2 for the PSN group (p-value $<0,05$ ), the same test reveals that there is no statistically reliable difference between Phase 2 and Phase 3 for the same group.

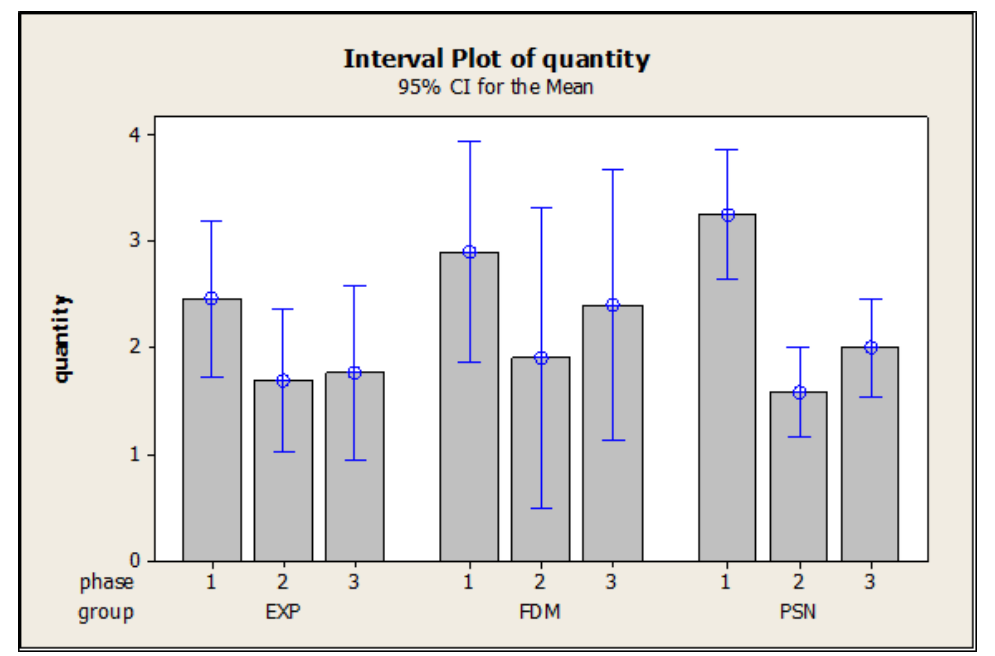

Figure 10. Mean number of ideas generated by students across the three phases.

Table 3. Differences and related p-values for t-test performed on the mean number of ideas generated.

\begin{tabular}{llll}
\hline Phase transition & Group & Difference & p-value \\
\hline \multirow{4}{*}{1 to 2} & EXP & $-0,79$ & 0,011 \\
& FDM & $-1,00$ & 0,096 \\
& PSN & $-1,67$ & 0,000 \\
\hline \multirow{3}{*}{2 to 3} & EXP & 0,08 & 0,820 \\
& FDM & 0,50 & 0,244 \\
& PSN & 0,417 & 0,054 \\
\hline
\end{tabular}

FDM group shows the highest mean growth for the same phase transition, but with a highly dispersive performance among students. However, it seems that the extension of the CI is mainly imputable to the subjects composing the group, as shown by results achieved for Phase 1 (i.e. before administering the investigated representations).

\subsection{Quality}

According to the judgment criteria illustrated above, all ideas were processed revealing that for all groups, QL remains almost unchanged among the three phases (Figure 11). The performed IRR test resulted in a Cohen's Kappa of 0,63 which means good concordance among the raters.

Since the observed differences were minimal, no additional statistical tests have been performed here. 


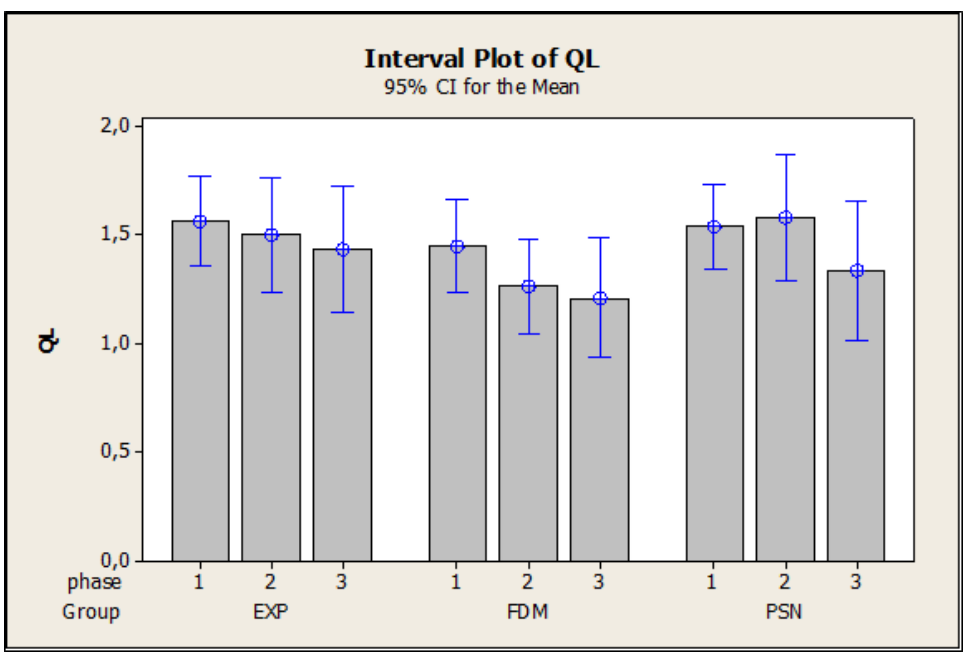

Figure 11. Mean quality scores observed across the three phases

Table 4. Quality differences registered across the three phases

\begin{tabular}{llll}
\hline Phase transition & Group & Difference & p-value \\
\hline \multirow{3}{*}{1 to 2} & EXP & $-0,63$ & 0,700 \\
& FDM & $-0,19$ & 0,220 \\
& PSN & 0,04 & 0,812 \\
2 to 3 & EXP & $-0,07$ & 0,730 \\
& FDM & $-0,06$ & 0,748 \\
& PSN & $-0,25$ & 0,246 \\
\hline
\end{tabular}

\subsection{Variety}

According to the variety metric introduced in Section 3, physical principles, working principles and (rough) embodiment characteristics have been extracted from the set of concepts proposed by students. The number of different observed items is listed in Table 5.

Table 5. Observed items variety.

\begin{tabular}{lcccc}
\hline Group & Item & Phase 1 & Phase 2 & Phase 3 \\
\hline \multirow{3}{*}{ EXP } & PP & 6 & 3 & 10 \\
& WP & 12 & 8 & 16 \\
& STR & 24 & 16 & 21 \\
FDM & PP & 6 & 6 & 11 \\
& WP & 12 & 10 & 23 \\
& STR & 17 & 15 & 22 \\
PSN & PP & 7 & 6 & 7 \\
& WP & 15 & 11 & 16 \\
& STR & 26 & 18 & 21 \\
\hline
\end{tabular}

The assessment of the variety level of the proposed ideas shows that the introduction of PSN and FDM representations brought non-negligible effects from Phase 1 to Phase 2. Indeed, differently with respect to 
the EXP group that didn't benefit from the introduction of the related supporting material, groups provided with FDM and PSN models recorded a similar positive variation (Figure 12 and Table 6). Differently, in Phase 3, EXP and FDM groups behaved quite similarly, while the PSN one revealed to be almost insensitive to the verbal stimulus.

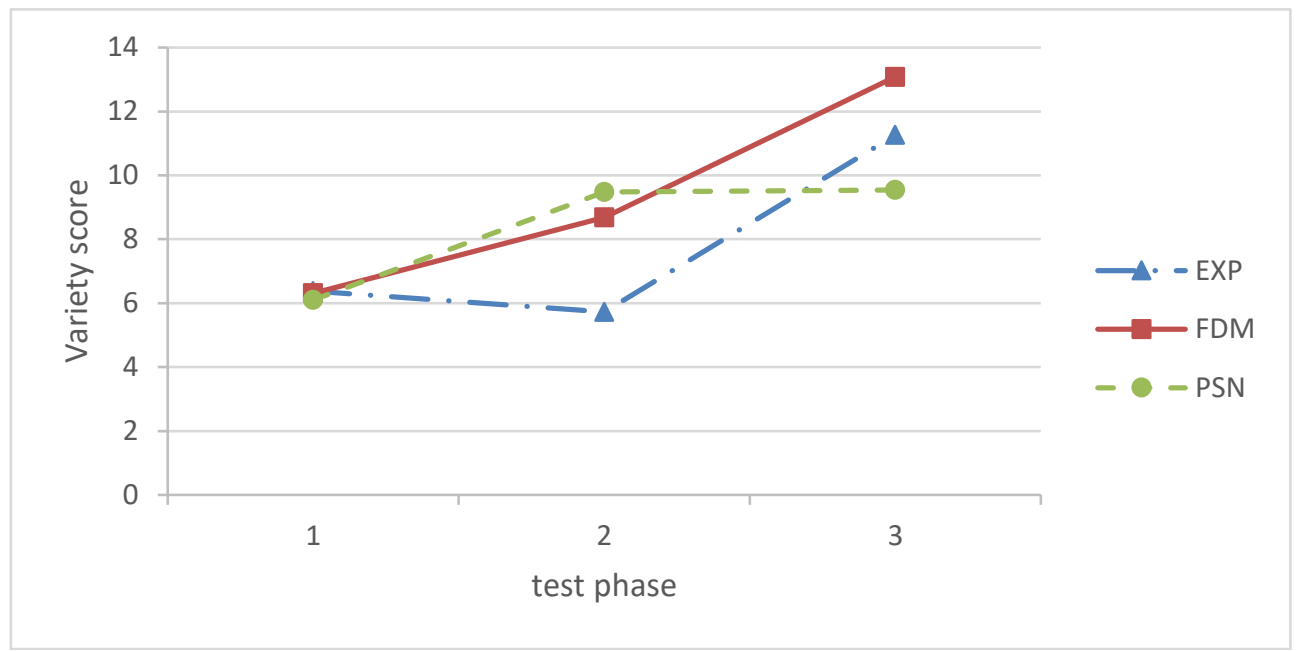

Figure 12. Variety observed for groups between the two test phases, according to (Shah et al., 2003)

Table 6. Variety differences for groups, between phase 1 and 2

\begin{tabular}{lcc}
\hline Phase transition & Group & Difference \\
\hline \multirow{3}{*}{1 to 2} & EXP & $-0,65$ \\
& FDM & 2,37 \\
& PSN & 3,37 \\
\hline \multirow{3}{*}{2 to 3} & EXP & 5,53 \\
& FDM & 4,40 \\
& PSN & 0,07 \\
\hline
\end{tabular}

\subsection{Novelty}

By applying the novelty metrics introduced in Section 3 on the sample of concepts produced by students, a significant heterogeneity has been observed (again) between groups. More precisely, as shown in Figure 13, the PSN group started with a quite higher novelty score. Nevertheless, the trend from the first to the second testing phase is practically the same for all the considered groups (see also Table 7), i.e. no statistically reliable difference can be observed between the first two phases. Differently, from the second to the third phase, all groups encountered a potentially statistically reliable (i.e. p-value $<0,05$ ) mean novelty growth. In this case, performed t-tests can be considered sufficiently reliable, because the analysis has been performed on novelty values of concepts conceived in each group, which are always higher than fifteen (i.e. the critical sample's extension below which the t-tests can be unreliable). Moreover, a verification of the Statistical Power of the particular conditions where statistically reliability has been observed (i.e. for EXP and FDM groups between Phase 2 and Phase 3), revealed values near or above 0,8. For the PSN group, the Statistical Power of the t-test was under 0,5, therefore an additional Mann-Whitney test has been performed between Phase 2 and Phase 3, revealing that no statistically reliable differences can be observed between the medians of the two phases. Differently, the same test executed on EXP and FDM groups reveals statistically reliable differences between Phase 2 and Phase 3. 


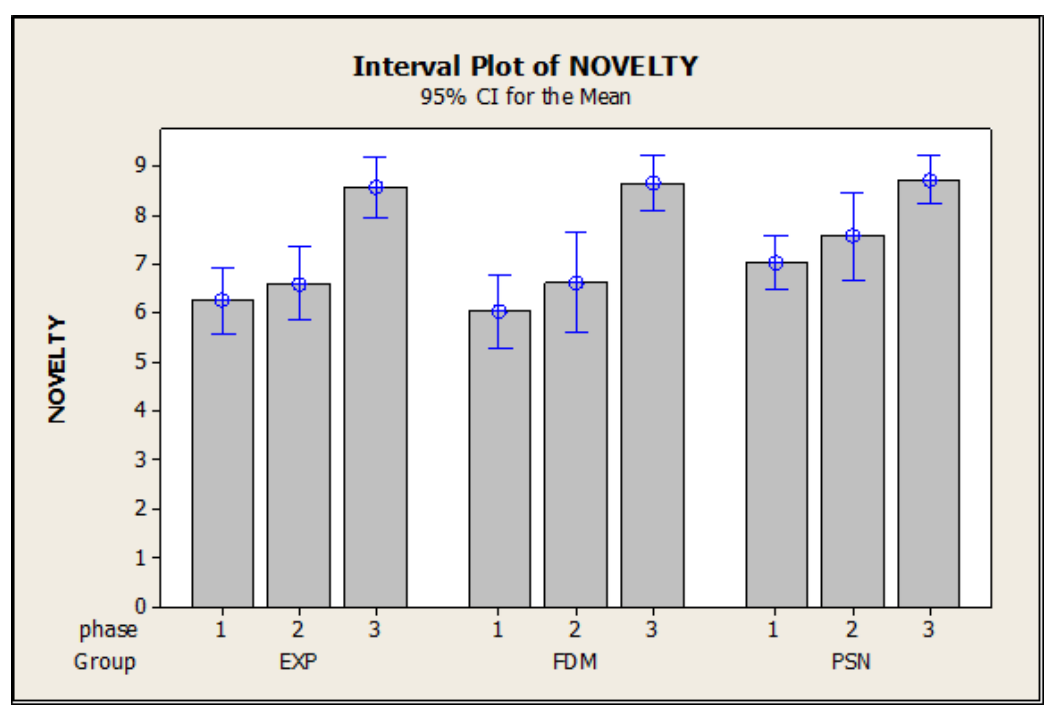

Figure 13. Mean novelty scores observed for groups between the two phases

Table 7. Differences and related p-values for t-test performed on the mean novelty observed across the three phases

\begin{tabular}{llll}
\hline Phase transition & Group & Difference & p-value \\
\hline \multirow{4}{*}{1 to 2} & EXP & 0,33 & 0,494 \\
& FDM & 0,58 & 0,352 \\
& PSN & 0,54 & 0,286 \\
2 to 3 & EXP & 1,98 & 0,000 \\
& FDM & 2,04 & 0,001 \\
& PSN & 1,15 & 0,025 \\
\hline
\end{tabular}

\subsection{Results analysis}

The results presented in this section show that the two examined representations have not brought any evident impact on QL quality scores. Even in terms of novelty, the investigated representations behaved quite similarly to the placebo, although the PSN registered a smaller and not statistically reliable growth of such a parameter from the second to the third phase.

Concerning Quantity, the PSN group shows a sensibly higher negative impact on Phase 2, while it is the sole bringing some potential positive effects on Phase 3. However, also FDM seems to potentially improve the quantity score in phase three, but the results are quite scattered.

The most evident effect has been registered in terms of variety of generated ideas (see Figure 12), since the introduction of FDM and PSN representations actually brought a similar positive effect in the second phase of the test. However, after the verbal suggestion provided in Phase 3, FDM behaved similarly to EXP, while PSN group was almost insensitive to the new stimuli. Such an evidence deserves to be investigated more in deep. In fact, it could mean that on the one hand, the considered abstract formalisms seem to bring positive effects in terms of variety but, on the other hand, maybe due to the nature of the provided verbal stimulus, it seems that some fixating effects somehow differently influence the two representation types. A possible interpretation of the observed difference is that the different representations could have influenced the design exploration paths pursued by students in a different manner, i.e. leading them to face the design task from different abstraction levels (e.g. searching for other behaviours instead of focusing on structural variants). 
According to these considerations, the following section deepens the analysis of the achieved data in order to gather more insights about the behaviour observed for Variety.

\section{Detailed study of the observed results}

To argue about the observed impact that FDM and PSN representations have on variety of conceived ideas, a further analysis has been conducted on the set of available data. More precisely, Variety has been re-assessed with an additional literature metric (Nelson, Wilson, Rosen, \& Yen, 2009), which is claimed to overcome some flaws of that previously adopted in this paper.

Moreover, to investigate around the different results observed in Phase 3, the explored design space has been analyzed more in deep by focusing on the actual effect of the verbal stimulus, and by the in-depth examination of the different abstraction levels considered by students when generating ideas.

\subsection{Confirming the observed Variety results}

To confirm the results observed with the metric of Shah and Vargas-hernandez (2003), the assessment has been repeated with the metric proposed by Nelson et al. (2009), which is claimed to avoid imprecise variety scores and to allow a measure of the actual design space exploration. In particular, this metric counts the differentiations at each item level (adapted here in PP, WP and STR), and does not operate the normalization of the variety scores on the quantity of ideas.

Achieved results are shown in Figure 14, where it is possible to observe that, although the trends are actually different, FDM and PSN still show a similar effect on Phase 2. More precisely, FDM and PSN representations somehow kept an almost constant Variety score from Phase 1 to Phase 2, while the EXP group encountered a quite evident reduction.

Concerning Phase 3, it is still possible to observe in Figure 14 that FDM behaves (positively) like the placebo and PSN preserves almost the same scores of the previous phase.

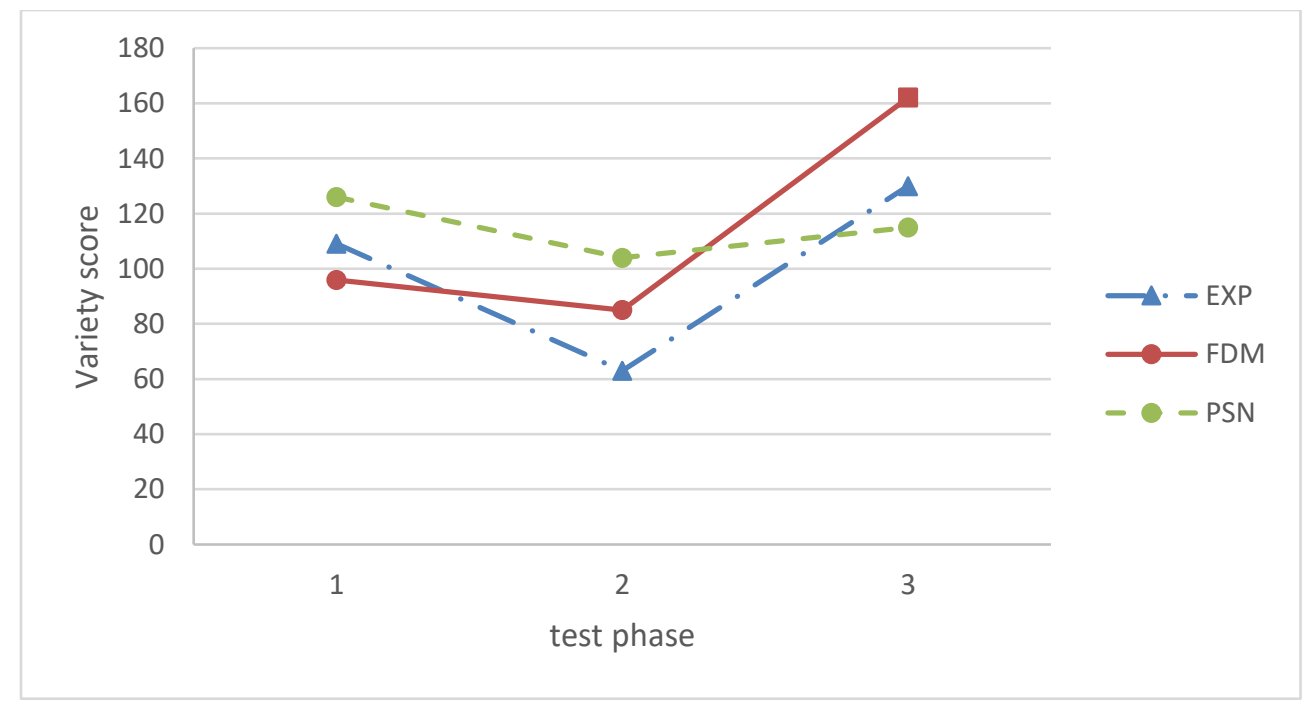

Figure 14. Variety observed for groups between the two test phases, according to (Nelson et al., 2009)

Thus, the countercheck operated with the additional metric confirms that the investigated representations (FDM and PSN) had a positive impact on Variety (in relation to the placebo) and that they led to different reactions to the verbal stimulus introduced in Phase 3. 


\subsection{Fixation on the "ball-tip"}

Design Fixation (Jansson \& Smith, 1991; Purcell \& Gero, 1996) constitutes a complex research argument, originally described in experimental psychology literature, and where many variables need to be taken under consideration (Vasconcelos \& Crilly, 2016). Moreover, it has been observed that different types of fixation exist, and different methods can be used (among which, the morphological analysis of Zwicky (1969)) to reduce its negative effects (Youmans \& Arciszewski, 2014). Therefore, any comprehensive consideration about design fixation should carefully consider many aspects, especially concerning the type of stimuli (Vasconcelos, Cardoso, Maria, Chen, \& Crilly, 2017).

The results shown in this paper about Variety and Novelty of design outcomes provide fundamental information about how the different representations impact on the design space exploration. However, more comprehensive assessments about design fixation should be performed by considering more theoretical aspects and structured procedures (Agogué et al., 2014; Crilly \& Cardoso, 2017; T. A. Nguyen \& Zeng, 2017).

Although a comprehensive analysis of design fixation is out of the scope of this paper, in reference to the detailed verbal stimulus provided in Phase 3, additional considerations can be argued about the different Variety behaviours. Accordingly, for each idea generated across the three phases, explicit references to the ball tip have been searched in the textual description and/or the related graphical representations in the sketches provided by students (see Figure 8). A sensible effect of the verbal stimulus in Phase 3 was naturally expected.

Figure 15 shows the percentage of ideas actually mentioning (graphically or textually) the use of the ball tip, together with the related confidence intervals. Similarly to the observed Variety scores (Figure 12), FDM and PSN representations averagely brought some effects in Phase 2, but with a quite dispersive behaviour (not statistically reliable). Moreover, no relevant differences among groups have been registered in Phase 3, since the investigated representations behaved quite similarly to the placebo. Therefore, the observed behaviour for variety in Phase 3 seems to be not directly dependent on the number of conceived solutions exploiting a "ball-tip".

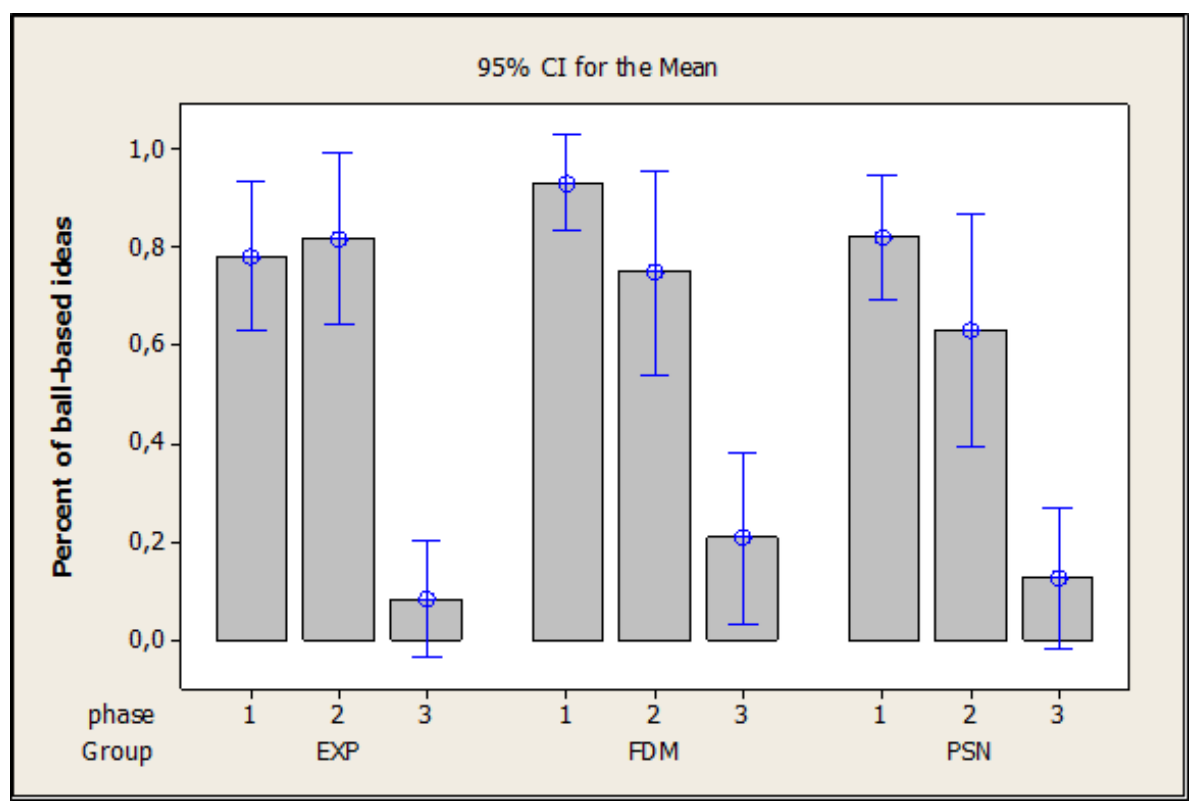

Figure 15. Percentage of the "ball-tip" ideas conceived by students across the three phases 


\subsection{Mapping the design space exploration}

Trying to shed further light on the behaviours that the two representations (FDM and PSN) show across the testing phases, the explored design space has been mapped by highlighting the abstraction levels considered by students for generating ideas different from the reference ball-tip pen. In this way, the authors aimed at identifying possible differences in the paths pursued by students when generating concepts. To that purpose, the PSN model of Figure 7 is considered as a reference since it provides different hierarchically organized levels of problems, whose solutions characterize the variety of concepts provided by students. Accordingly, by analyzing the sketches and the related textual descriptions, the following four recurrent levels of abstraction have been identified, to be used for the identification of the level at which the analyzed concepts differ:

- L1: Identification of principles for impressing symbols on paper sheets (e.g. concepts exploiting alternatives to fluid ink).

- L2: This level contains solutions based on fluid ink, but using different ways for its deposition on a paper sheet (e.g. concepts exploiting fluid ink, but with alternatives to a liquid flow directed to the paper surface).

- L3: This level contains solutions based on liquid ink directed to the paper surface, but using different solutions to fulfil secondary needs (e.g. alternative solutions for storing, feeding and/or directing the liquid ink) .

- L4: Solutions related to further details of the same pen type shown in the task description Figure 5 (i.e. alternatives to structural details of a classical ball-point tip).

It is possible to consider the proposed analysis framework as a particular derivation of the well-known Function-Behavior- Structure (FBS) formalism (Gero \& Kanessegiesser, 2004; Vermaas \& Dorst, 2007), which can be also used for identifying different abstraction levels (Sarkar \& Chakrabarti, 2011). Indeed, levels from L1 to L3 are a sort of detailed Behavior sub-levels (problems concerning how the system perform the main function), while L4 concerns Structure details.

To provide an example, Table 8 reports the identified abstraction levels characterizing the two concepts shown in Figure 8.

Table 8. Abstraction levels of the problems faced for generating the concepts represented in Figure 8.

\begin{tabular}{lll}
\hline Concept & $\begin{array}{l}\text { Abstraction } \\
\text { level }\end{array}$ & Description \\
\hline a) & L3 & $\begin{array}{l}\text { The main problem faced by the designer concerns an alternative } \\
\text { principle for feeding the liquid ink (spring force instead of } \\
\text { gravity). }\end{array}$ \\
b) & L1 & $\begin{array}{l}\text { In this solution, the designer proposes an alternative to the ink } \\
\text { for impressing symbols on a paper sheet. }\end{array}$ \\
\hline
\end{tabular}

Then, according to the examples shown in Table 8, the main abstraction levels for all the concepts generated by students have been identified. More precisely, similarly to the Variety's item assignment, the authors assigned these "differentiation" levels through a conjoint analysis session. The obtained results are shown in Figure 16. 

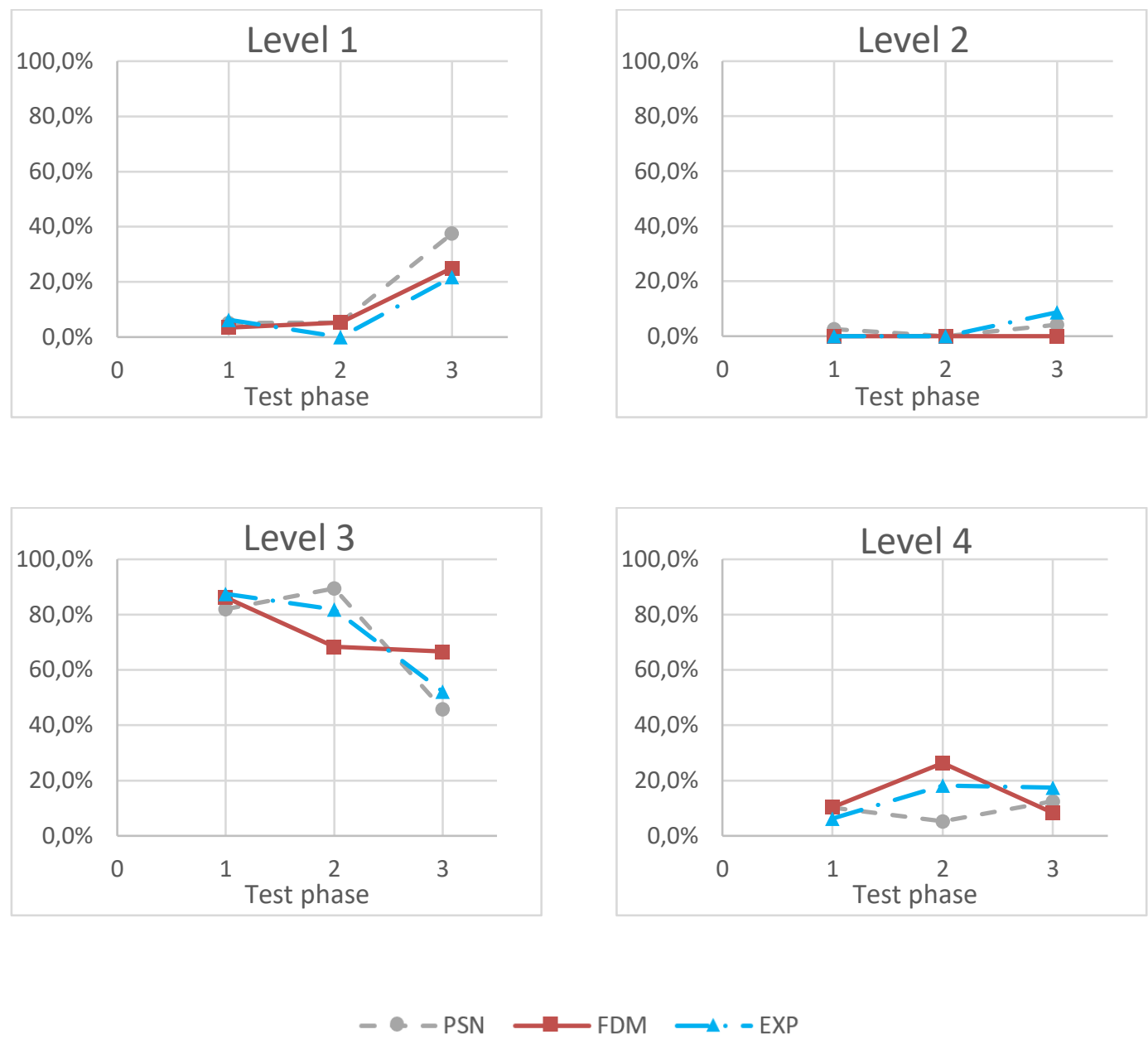

Figure 16. Percentages of concepts differing from the reference one at each of the four considered levels.

In reference to the results shown in Figure 16, it is possible to observe that the three groups behaved quite similarly in terms of alternative solutions at Level 1. More precisely, a slightly superior increment can be observed for the PSN group in Phase 3, and EXP group neglected Level 1 in Phase 2. FDM neglected Level 2 across the three phases, while the PSN group proposes few solutions at this level only in Phase 3 and Phase 1, while EXP only in Phase 3. The most evident impact of the different representations in terms of explored levels is shown for L3, which is the most explored one. Indeed, while the three groups start almost equally from Phase 1, in Phase 2 PSN and EXP substantially maintained the same percentage of solutions (within a range of $10 \%$ ) while the FDM group encountered a reduction of about $20 \%$. These differences persist also in Phase 3, where the FDM group is almost insensitive to the verbal stimulus, while EXP and PSN groups had a reduction of about $40 \%$ of solutions at this level. Concerning Level 4, the PSN group behaved quite differently from EXP and FDM in Phase 2. Moreover, FDM showed a reduction of L4 solutions in Phase 3.

Therefore, Figure 16 shows that in Phase 2, the effects of FDM and EXP in terms of explored abstraction levels are quite similar, while PSN behaved differently. At a glance, no evident correlation exists between explored abstraction levels and Variety scores observed in Phase 2 (where, instead, a similar behaviour was observed between PSN and FDM). Especially observing Levels 3 and 4, the effect of the verbal stimulus 
(Phase 3) for FDM was quite different from that registered for PSN and EXP, thus no evident correlations can be identified with the Variety scores observed in Phase 3.

Nevertheless, although the observed differences cannot be used to explain the different Variety scores registered across the three phases, it is possible to observe that different representations led to different idea generation and problem-solving paths.

\section{Discussion}

The results presented in Section 4 and those coming from the further investigations described in Section 5 allow formulating an answer to the research question introduced in this paper. Nevertheless, some limits can be ascribed to this work, thus leading to new potential research hints.

\subsection{Answer to the research question}

Concerning Quantity, it has been shown that the introduction of the investigated representations led to negative effects, especially for the PSN one. This might be explained with the time necessary to interpret the information provided, since the subjects were unlearned about the FDM and the PSN formalism.

Concerning Quality of solutions (according to the adopted metric), no reliable differences have been observed between the three groups. This means that those design representations don't provide the subjects with information which is outside their background knowledge. On the contrary, subjects of all groups have the same capability to judge the viability of a solution independently from the received information set. Clearly, this result might be a consequent of the design task proposed in this experiment. A design task related to a less-common product and/or based on a more complex functioning mechanism, might bring to different utcomes.

Similarly, also in terms of Novelty it has not been possible to observe evident advantages.

Some positive effects have been observed in terms of Variety, because the introduction of the PSN and FDM representations in Phase 2 led to positive differences in relation to the placebo (Figure 12 and Figure 14). More precisely, the introduction of both PSN and FDM representations in Phase 2 led students to generate more solutions that sensibly differ from the ball-tip structural detail (Figure 15), leading to a positive impact (in relation to the EXP group) on Variety in Phase 2 (Figure 12 and Figure 14). This is in accordance with the outcomes observed by Howard, Culley, \& Dekoninck (2011), i.e. more ambiguous (intended here as abstract) stimuli tend to be less fixating.

However, the in-depth analysis of the abstraction levels considered by students revealed that, generally, the introduction of FDM in Phase 2 led to a higher interest toward structural details (Level 4 in Figure 16). It means that notwithstanding the similar positive effects on Variety in Phase 2, PSN and FDM representations differently influenced the student's design path. Accordingly, also the introduction of the verbal stimulus highlighted non-negligible differences between the Variety scores reached by the two groups (FDM and PSN) in Phase 3 (Figure 16), especially at Level 3.

In a few words, the work presented in this paper gave a first response to the research question, highlighting the positive effects of the considered representations on the observed on Variety scores. Nevertheless, it also highlights that the two investigated representations led students to different idea generation paths.

\subsection{Further research hints}

The first research clue directly related to the observed results concerns the need to shed light on the cognitive mechanisms behind the different reactions observed for FDM and PSN. It could be done, for example, by performing additional experiments with different tasks and different samples, in order to observe the different abstraction levels pursued by designers for generating ideas, and then to check for recurring differences among the investigated representations. A further motivation to the need of collecting 
additional data resides in the statistical reliability of the assessments performed here. Indeed, the limited sample considered in this work should be widened to extract valid and more reliable evidence.

Other limitations can be ascribed to this work, each of them potentially leading to further research hints. Indeed, the representation of multiple concept variants was intentionally neglected (see Section 2.3), thus neglecting any investigation of the effects of the different ways for storing and sharing information about more solutions. The results shown in this paper put the basis and constitute a reference for this kind of research, highlighting the effects directly imputable to the different schematizations of a single solution.

Another limitation of this work, which can constitute a trigger for future research, is the lack of feedback from students about the efforts needed for performing the required task. This kind of data could be important for the identification of a "comfortable" but comprehensive way for eliciting information from previously performed design tasks. Standard forms could be used for administering surveys to that purpose, e.g. the NASA Task Load Index (S. G. Hart, 2006; Sandra G. Hart \& Staveland, 1988) or some specific variants but more advanced analysis can be performed also with design protocol analysis and/or with empirical approaches (e.g. P. Nguyen, Nguyen, \& Zeng (2018)).

Moreover, the investigated representations contain a variable quantity of textual and pictorial information. Literature highlighted some differences among the effects of different ways to provide design information, (Atilola et al., 2015; Borgianni, Rotini, \& Tomassini, 2017; Cheng, Mugge, \& Schoormans, 2014; Ekwaro-Osire, Cruz-Lozano, Endeshaw, \& Dias, 2016; Goldschmidt \& Sever, 2011), therefore it is possible to infer that structuring the same representations with different amounts/quality of text and images could differently influence the design outcomes. Consequently, future research should be performed on this specific topic, aiming at the identification of the most effective way to provide design information about information generated in previous conceptual design tasks.

Furthermore, the results presented in this paper could potentially pave the way for new tests with samples of designers capable to apply not only the formalisms required for design representations but also the methods' rationale. In this way, by comparing results from experiments with different samples of designers (learned VS un-learned), it could be possible to argue about the effects directly imputable to representations and those imputable to the design rationale imposed by the method itself.

\section{Conclusions}

The objective of the work described in this paper was to investigate about the existence of any creative advantage given by the availability of design representations from earlier conceptual design activities while performing an individual conceptual design task. The reasons behind this research question reside in the importance of reusing information achieved in the fuzzy front end of the design process, and on the actual difficulties in sharing it by means of textual/graphical technical reports. Authors inferred that representations from systematic conceptual design methods could be a valid tool to comprehensively and schematically share this kind of information. However, the authors acknowledged that due to the scarce diffusion of these methods in industry, it is quite probable that part of involved designers have not a sufficient knowledge about the method from which representation originated. For this reason, the test has been performed in a conservative way, by considering a sample constituted by engineering students unlearned about systematic design methods.

The experiment was structured in three sequential design sessions, and students were subdivided into three groups, in order to evaluate the effects of the considered conceptual design representations in reference to a sort of placebo, i.e. a third representation type (a standard drawing) normally used in technical reports.

The investigation procedure foresaw the application of well-known literature metrics for evaluating the effectiveness of idea generation sessions, but also further in-depth analyses have been conducted. Indeed, a positive effect has been observed in terms of Variety of concepts devised by students. However, the third test phase highlighted different behaviours between the two investigated representations. These differences led the authors to the mentioned additional analyses, which highlighted that although the two considered representations potentially allow performing more deepened design space explorations, the different 
behaviours observed in the third phase are quite complex and cannot be explained here. Moreover, it also emerged that notwithstanding the similar positive effect in terms of Variety, the two representations led students to follow quite different design paths.

Therefore, the results shown in this paper provide a first evidence about the potential usefulness of conceptual design representations in reusing information from previous design activities but also highlight that the cognitive mechanism behind the observed effects is not trivial to understand. Accordingly, additional research is needed and this paper constitutes a reference for researchers interested in this specific argument, also providing several research hints for future activities.

\section{Author Biographies}

G. Cascini holds a Ph.D. in Machine Design and is Full Professor at Politecnico di Milano, Dept. of Mechanical Engineering. His research interests cover Design Methods and Tools with a focus on the concept generation stages both for product and process innovation. He is member of the Board of Management of the Design Society. He has coordinated several research projects, among the others the European Project Marie Curie-IAPP FORMAT (FORecast and Roadmapping for MAnufacturing Technologies). Currently he is the coordinator of the European projects: SPARK: Spatial Augmented Reality as a Key for co-creativity (Horizon 2020 - ICT) and OIPEC: Open innovation Platform for university-Enterprise Collaboration: new product, business and human capital development (Erasmus+ Capacity Building in Higher Education). He has authored more than 140 papers presented at International Conferences and published in authoritative Journals and 13 patents.

L. Fiorineschi took the Master Degree in Mechanical Engineering at the University of Florence, Italy (2006) and the Ph.D. in Industrial Engineering at the same Institution (2015). He is currently Research Assistant at the Industrial Engineering Department of the University of Florence, Italy. His research interests include engineering Design Methods, Product Architecture, Prototyping and Intellectual Property. He authored scientific papers presented in International Conferences and published in authoritative Journals, one technical handbook and one patent. He actively participated to several multidisciplinary projects and collaborated to design-related courses at Florence University.

F. Rotini took the master degree in Mechanical Engineering at Florence University, Italy. He is $\mathrm{Ph} . \mathrm{D}$. in Machine Design and works as Associate Professor since 2017 at the School of Engineering of the Florence University. His main topics of research concern the development of methodologies to support Creativity in product and process innovation, Business Process Re-engineering activities and the Product Design Cycle. He is author of more than 90 scientific papers presented in International Conferences and published in authoritative Journals, one book and three patents. He is involved in many research projects having national and international relevance. He holds courses at the Schools of Engineering and Architecture, both belonging to Florence University. 


\section{Appendix}

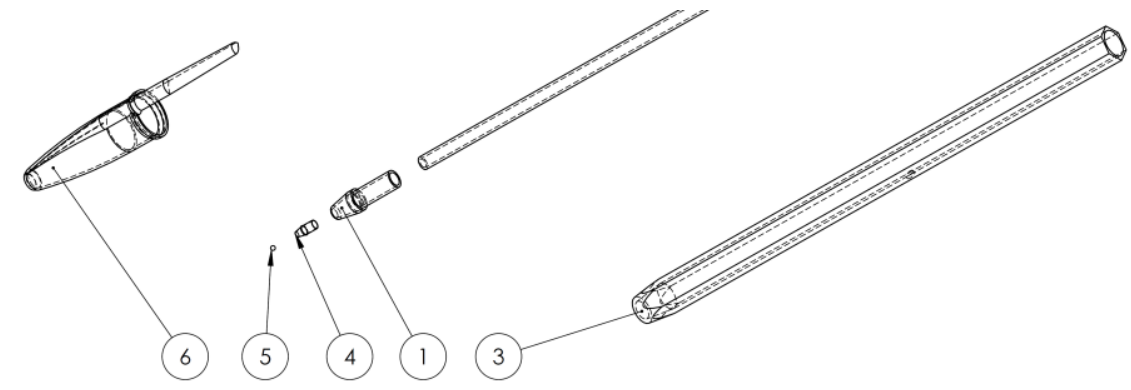

\begin{tabular}{|c|l|c|c|}
\hline Number & \multicolumn{1}{|c|}{ Name } & Material & Quantity \\
\hline 1 & lower support & plastic & 1 \\
\hline 2 & ink tube & plastic & 1 \\
\hline 3 & external tube & transparent plastic & 1 \\
\hline 4 & ball support & brass & 1 \\
\hline 5 & ball & tungsten carbide & 1 \\
\hline 6 & cap & plastic & 1 \\
\hline 7 & back cap & plastic & 1 \\
\hline
\end{tabular}

Figure 17. Representation provided to the EXP group in Phase 2 


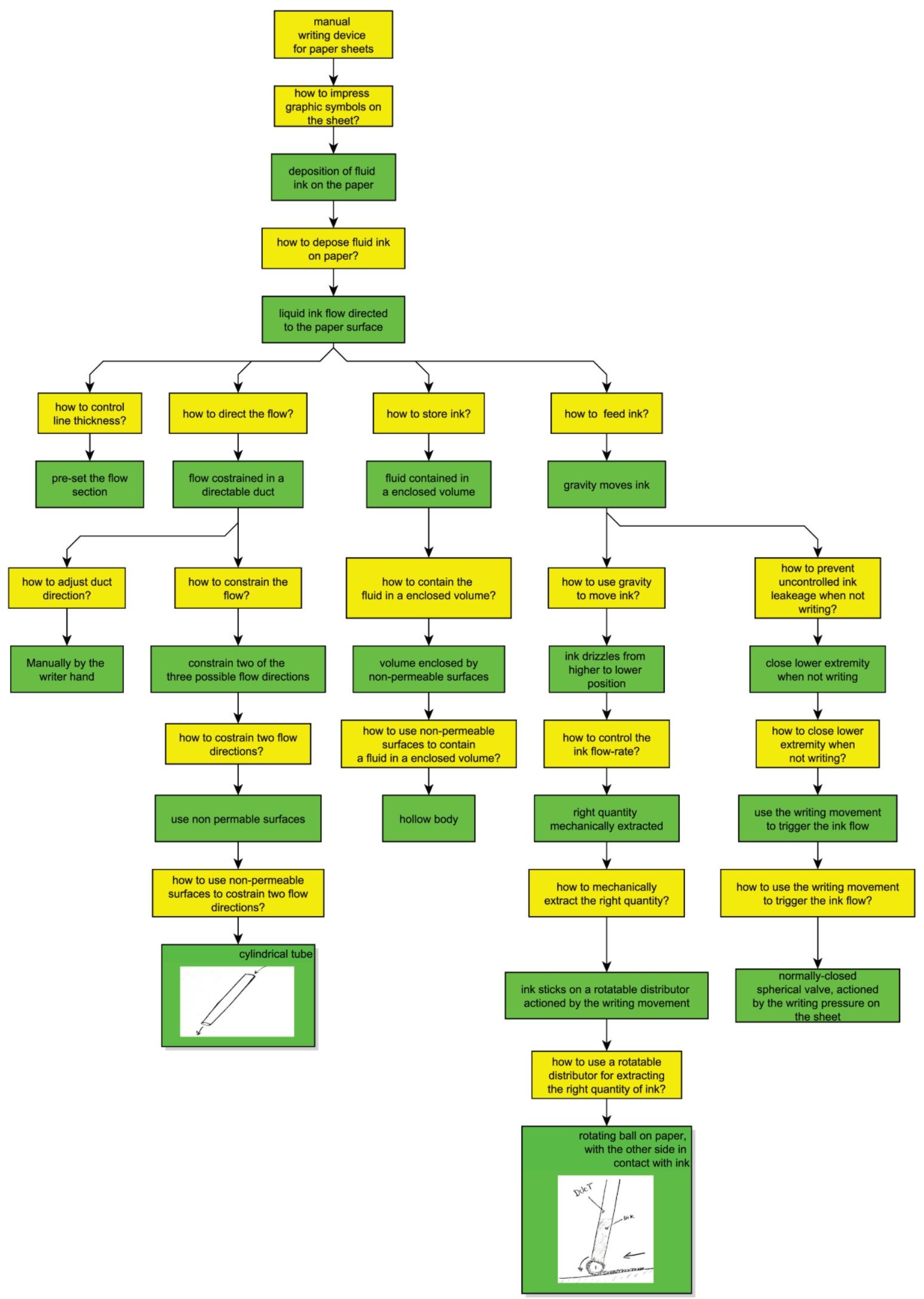

Figure 18. Representation provided to the PSN group in Phase 2 

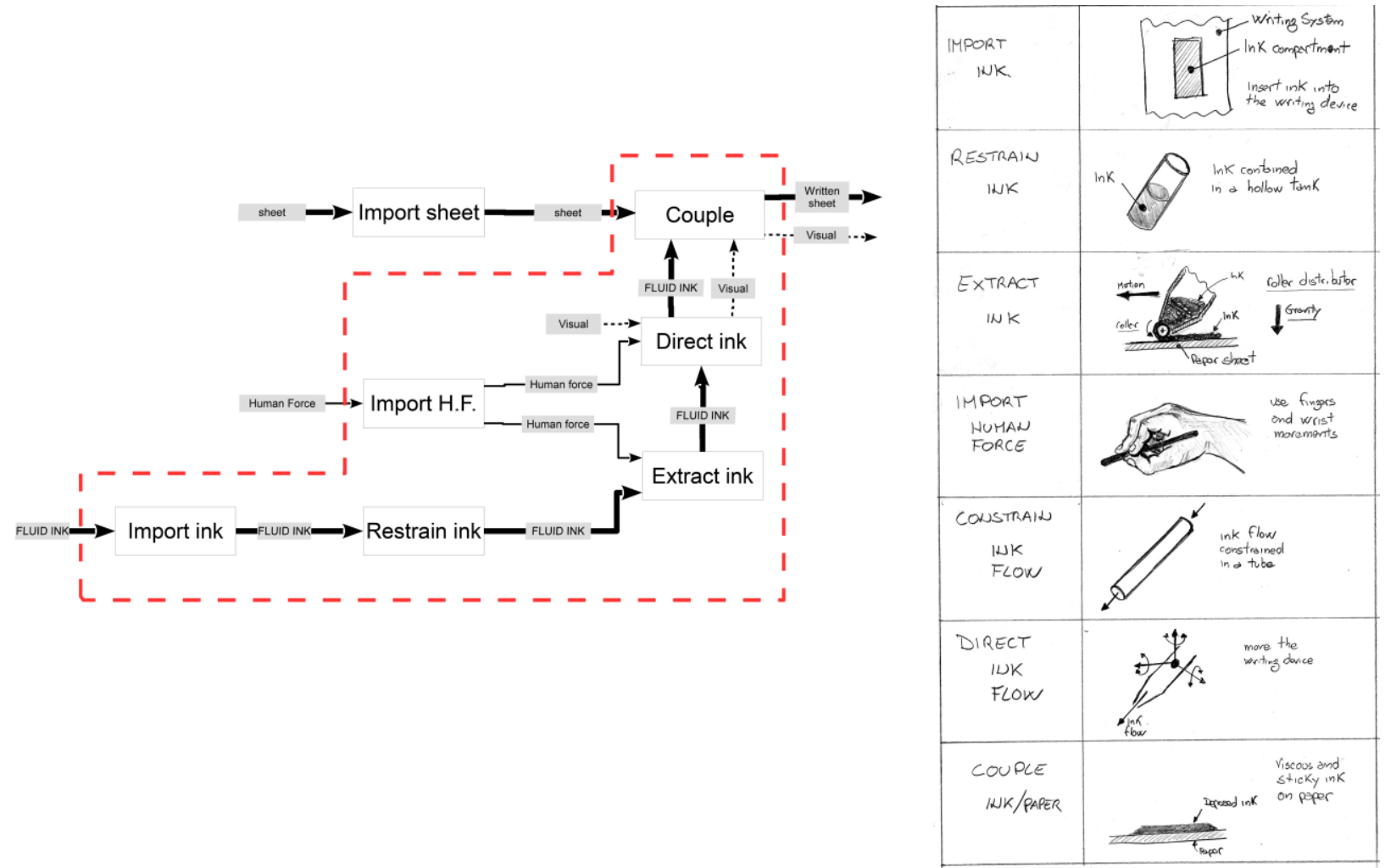

Figure 19. Representation provided to the FDM group in Phase 2

\section{Reference list}

Agogué, M., Kazakçi, A., Hatchuel, A., Le Masson, P., Weil, B., Poirel, N., \& Cassotti, M. (2014). The impact of type of examples on originality: Explaining fixation and stimulation effects. Journal of Creative Behavior, 48(1), 1-12. https://doi.org/10.1002/jocb.37

Ahmad, N., Wynn, D. C., \& Clarkson, P. J. (2013). Change impact on a product and its redesign process: A tool for knowledge capture and reuse. Research in Engineering Design, 24(3), 219-244. https://doi.org/10.1007/s00163-012-0139-8

Ahmed, S. (2005). Encouraging reuse of design knowledge: A method to index knowledge. Design Studies, 26(6), 565-592. https://doi.org/10.1016/j.destud.2005.02.005

Atilola, O., \& Linsey, J. (2015). Representing analogies to influence fixation and creativity: A study comparing computer-aided design, photographs, and sketches. Artificial Intelligence for Engineering Design, Analysis and Manufacturing, 29(02), 161-171. https://doi.org/10.1017/S0890060415000049

Atilola, O., Tomko, M., \& Linsey, J. S. (2015). The effects of representation on idea generation and design fixation: A study comparing sketches and function trees. Design Studies, 42, 110-136. https://doi.org/10.1016/j.destud.2015.10.005

Baxter, D., Gao, J., Case, K., Harding, J., Young, B., Cochrane, S., \& Dani, S. (2007). An engineering design knowledge reuse methodology using process modelling. Research in Engineering Design, 18(1), 37-48. https://doi.org/10.1007/s00163-007-0028-8 
Borgianni, Y., Rotini, F., \& Tomassini, M. (2017). Fostering ideation in the very early design phases: How textual, pictorial and combined stimuli affect creativity. ICED17: 21st International Conference on Engineering Design, 8(DS87-8), 139-148.

Chakrabarti, A., \& Bligh, T. P. (2001). A scheme for functional reasoning in conceptual design. Design Studies, 22, 493-517. https://doi.org/10.1016/S0142-694X(01)00008-4

Chandrasegaran, S. K., Ramani, K., Sriram, R. D., Horváth, I., Bernard, A., Harik, R. F., \& Gao, W. (2013). The evolution, challenges, and future of knowledge representation in product design systems. Computer-Aided Design, 45(2), 204-228. https://doi.org/10.1016/j.cad.2012.08.006

Cheng, P., Mugge, R., \& Schoormans, J. P. L. (2014). A new strategy to reduce design fixation: Presenting partial photographs to designers. Design Studies, 35(4), 374-391. https://doi.org/10.1016/j.destud.2014.02.004

Crilly, N., \& Cardoso, C. (2017). Where next for research on fixation, inspiration and creativity in design? Design Studies, 50, 1-38. https://doi.org/10.1016/j.destud.2017.02.001

Eckert, C. (2013). That which is not form: The practical challenges in using functional concepts in design. Artificial Intelligence for Engineering Design, Analysis and Manufacturing, 27(03), 217-231. https://doi.org/10.1017/S089006041300022X

Eckert, C., Alink, T., \& Ruckpaul, A. (2011). Different notions of function : results from an experiment on the analysis of an existing product. Journal of Engineering Design, 22(11-12), 811-837. https://doi.org/http://dx.doi.org/10.1080/09544828.2011.603297

Eder, W., \& Hosnedl, S. (2008). Design Engineering - A Manual for Enhanced Creativity -. CRC Press.

Eisenbart, B., Gericke, K., \& Blessing, L. T. M. (2013). An analysis of functional modeling approaches across disciplines. Artificial Intelligence for Engineering Design, Analysis and Manufacturing, 27(03), 281-289. https://doi.org/10.1017/S0890060413000280

Ekwaro-Osire, S., Cruz-Lozano, R., Endeshaw, H. B., \& Dias, J. P. (2016). Uncertainty in Communication with a Sketch. Journal of Integrated Design and Process Science, 20(4), 43-60. https://doi.org/10.3233/jid-2016-0022

Eppinger, S. D., \& Ulrich, K. T. (2007). Product Design and Development. Mc Graw HIll.

Fiorineschi, L., Frillici, F. S., \& Rotini, F. (2018). A-Posteriori Novelty Assessments for Sequential Design Sessions. In International Design Conference - Design 2018 (pp. 1079-1090).

Fiorineschi, L., Rotini, F., \& Rissone, P. (2016). A new conceptual design approach for overcoming the flaws of functional decomposition and morphology. Journal of Engineering Design, 27(7). https://doi.org/10.1080/09544828.2016.1160275

Gero, J. S., \& Kanessegiesser, U. (2004). The situated function-behaviour-structure framework.pdf. Design Studies, 25(4), 373-391.

Goldschmidt, G., \& Sever, A. L. (2011). Inspiring design ideas with texts. Design Studies, 32(2), 139-155. https://doi.org/10.1016/j.destud.2010.09.006

Gonçalves, M., Cardoso, C., \& Badke-Schaub, P. (2014). What inspires designers? Preferences on inspirational approaches during idea generation. Design Studies, 35(1), 29-53. https://doi.org/10.1016/j.destud.2013.09.001

Hart, S. G. (2006). Nasa-Task Load Index (NASA-TLX); 20 Years Later. Proceedings of the Human Factors and Ergonomics Society Annual Meeting, 50(9), 904-908. https://doi.org/10.1177/154193120605000909

Hart, S. G., \& Staveland, L. E. (1988). Development of NASA-TLX (Task Load Index): Results of Empirical and Theoretical Research. Advances in Psychology, 52(C), 139-183. https://doi.org/10.1016/S0166-4115(08)62386-9

Heller, J. E., Schmid, A., Löwer, M., \& Feldhusen, J. (2014). The Dilemma of Morphological Analysis in 
Product Concept Synthesis - New Approaches for Industry and Academia. In International Conference on Engineering Design - DESIGN 2014 (pp. 201-210).

Howard, T. J., Culley, S., \& Dekoninck, E. a. (2011). Reuse of ideas and concepts for creative stimuli in engineering design. Journal of Engineering Design, 22(8), 565-581. https://doi.org/10.1080/09544821003598573

Howard, T. J., Dekoninck, E. A., \& Culley, S. J. (2010). The use of creative stimuli at early stages of industrial product innovation. Research in Engineering Design, 21(4), 263-274. https://doi.org/10.1007/s00163-010-0091-4

Jansson, D. G., \& Smith, S. M. (1991). Design fixation. Design Studies, 12(1), 3-11. https://doi.org/10.1016/0142-694X(91)90003-F

Johnson, T. A., Caldwell, B. W., Cheeley, A., \& Green, M. G. (2016). Comparison and Extension of Novelty Metrics for Problem-Solving Tasks. In Proceedings of the ASME 2016 International Design Engineering Technical Conferences and Computers and Information in Engineering Conference IDETC/CIE 2016. https://doi.org/10.1115/DETC2016-60319

Kroll, E. (2013). Design theory and conceptual design: contrasting functional decomposition and morphology with parameter analysis. Research in Engineering Design, 165-183. https://doi.org/10.1007/s00163-012-0149-6

Linsey, J. S., Clauss, E. F., Kurtoglu, T., Murphy, J. T., Wood, K. L., \& Markman, a. B. (2011). An Experimental Study of Group Idea Generation Techniques: Understanding the Roles of Idea Representation and Viewing Methods. Journal of Mechanical Design, 133(3), 031008. https://doi.org/10.1115/1.4003498

Linsey, J. S., Wood, K. L., \& Markman, A. B. (2008). Modality and representation in analogy. Ai Edam, 22(02), 85-100. https://doi.org/10.1017/S0890060408000061

Medyna, G., Nonsiri, S., Coatanéa, E., \& Bernardb, A. (2012). Modelling, evaluation and simulation during the early design stages: Toward the development of an approach limiting the need for specific knowledge. Journal of Integrated Design and Process Science, 16(3), 111-131. https://doi.org/10.3233/jid-2012-0006

Nelson, B. A., Wilson, J. O., Rosen, D., \& Yen, J. (2009). Refined metrics for measuring ideation effectiveness. Design Studies, 30(6), 737-743. https://doi.org/10.1016/j.destud.2009.07.002

Nguyen, P., Nguyen, T. A., \& Zeng, Y. (2018). Empirical approaches to quantifying effort, fatigue and concentration in the conceptual design process: An EEG study. Research in Engineering Design, 29(3), 393-409. https://doi.org/10.1007/s00163-017-0273-4

Nguyen, T. A., \& Zeng, Y. (2017). A theoretical model of design fixation. International Journal of Design Creativity and Innovation, 5(3-4), 185-204. https://doi.org/10.1080/21650349.2016.1207566

Pahl, G., Beitz, W., Feldhusen, J., \& Grote, K. H. (2007). Engineering design 3rd ed. Springer-Verlag London. https://doi.org/https://doi.org/10.1007/978-1-84628-319-2

Peeters, J., Verhaegen, P. A., Vandevenne, D., \& Duflou, J. R. (2010). Refined Metrics for Measuring Novelty in Ideation. In Proceedings of IDMME - Virtual Concept 2010 (pp. 1-4).

Pugh, S. (1991). Total Design. Integrated Methods for Succesfull Product Engineering. Reading, Massachusetts: Addison Wesley Publishing Company.

Purcell, A. T., \& Gero, J. S. (1996). Design and other types of fixation. Design Studies, 17(4 SPEC. ISS.), 363-383. https://doi.org/10.1016/S0142-694X(96)00023-3

Qin, H., Wang, H., \& Johnson, A. L. (2017). A RFBSE model for capturing engineers' useful knowledge and experience during the design process. Robotics and Computer-Integrated Manufacturing, 44, 3043. https://doi.org/10.1016/j.rcim.2016.08.004

Ross, S. M. (2009). Probability and Statistics for Engineers and Scientists (4th ed.). Elsevier Academic Press. Retrieved from http://linkinghub.elsevier.com/retrieve/pii/B9780123704832000114 
Sarkar, P., \& Chakrabarti, A. (2011). Assessing design creativity. Design Studies, 32(4), 348-383. https://doi.org/10.1016/j.destud.2011.01.002

Shah, J. J., Vargas-Hernandez, N., \& Smith, S. M. (2003). Metrics for measuring ideation effectiveness. Design Studies, 24(2), 111-134. https://doi.org/10.1016/S0142-694X(02)00034-0

Sheskin, D. J. (2003). Handbook of parametric and nonparametric statistical procedures. CRC Press.

Tomiyama, T., Gu, P., Jin, Y., Lutters, D., Kind, C., \& Kimura, F. (2009). Design methodologies: Industrial and educational applications. CIRP Annals, 58(2), 543-565. https://doi.org/10.1016/j.cirp.2009.09.003

Ullman, D. G. (2010). The Mechanical Design Process 4th ed. New York, USA: Mc Graw HIll.

Vargas-Hernandez, N., Okudan, G. E., \& Schmidt, L. C. (2012). Effectiveness Metrics for Ideation: Merging Genealogy Trees and Improving Novelty Metric. In Proceedings of the ASME 2012 International Design Engineering Technical Conferences \& Computers and Information in Engineering Conference IDETC/CIE 2012. https://doi.org/10.1115/DETC2012-70295

Vasconcelos, L. A., Cardoso, C. C., Maria, S., Chen, C., \& Crilly, N. (2017). Inspiration and Fixation: The Influences of Example Designs and System Properties in Idea Generation. Journal of Mechanical Design, 139(March), 1-13. https://doi.org/10.1115/1.4035540

Vasconcelos, L. A., \& Crilly, N. (2016). Inspiration and fixation: Questions, methods, findings, and challenges. Design Studies, 42, 1-32. https://doi.org/10.1016/j.destud.2015.11.001

Vermaas, P. E., \& Dorst, K. (2007). On the conceptual framework of John Gero's FBS-model and the prescriptive aims of design methodology. Design Studies, 28, 133-157. https://doi.org/10.1016/j.destud.2006.11.001

Vermaas, P. E., \& Eckert, C. (2013). My functional description is better! Artificial Intelligence for Engineering Design, Analysis and Manufacturing, 27(03), 187-190. https://doi.org/10.1017/S089006041300019X

Youmans, R. J., \& Arciszewski, T. (2014). Design fixation: Classifications and modern methods of prevention. Artificial Intelligence for Engineering Design, Analysis and Manufacturing, 28(02), 129137. https://doi.org/10.1017/S0890060414000043

Zwicky, F. (1969). Discovery, Invention, Research-Through the Morphological Approach. New York: Macmillan Publisher. 